\title{
Comprensión del Sistema de los Números Complejos: Un Estudio de Caso a Nivel Escolar y Universitario
}

\author{
Valeria N. Randolph* y Marcela C. Parraguez \\ Instituto de Matemáticas, Facultad de Ciencias, Pontificia Universidad Católica de Valparaíso, Blanco Viel № 596, Cerro \\ Barón, Valparaíso, Chile. (e-mail: valeria.randolph.v@mail.pucv.cl; marcela.parraguez@pucv.cl) \\ * Autor a quien debe ser dirigida la correspondencia
}

Recibido Mar. 5, 2019; Aceptado Abr. 29, 2019; Versión final Jun. 27, 2019, Publicado Dic. 2019

\begin{abstract}
Resumen
Se reporta una investigación sobre cómo estudiantes de educación escolar y universitaria comprenden el Sistema de los Números Complejos y sobre cómo es posible alcanzar la comprensión profunda de éste. Desde un enfoque cognitivo, se utiliza la teoría de los Modos de Pensamiento que permite, mediante sus elementos, situar tres modos de pensar el objeto matemático: el modo Sintético-Geométrico, el modo Analítico-Aritmético y el modo Analítico-Estructural. A partir de un estudio histórico-epistemológico y matemático, se caracterizan los tres modos de pensar el sistema numérico y se aplican dos cuestionarios de actividades matemáticas a cinco casos de estudio. El análisis de las producciones de los estudiantes da cuenta de una falta de articulación de los modos de pensamiento, privilegiando el modo Analítico-Aritmético y careciendo de tránsitos hacia los otros dos modos. Esto permite concluir que existe una comprensión fragmentada del objeto y que es necesario potenciar el trabajo desde el punto de vista geométrico y estructural.
\end{abstract}

Palabras clave: números complejos; sistema numérico; modos de pensamiento; comprensión de los números; articuladores del pensamiento

\section{Understanding the Complex Number System: A Case Study in High School and University Level}

\begin{abstract}
The results of an investigation on how students from school education and university education understand the Complex Number System and how it is possible to achieve a deep understanding of it is reported. From a cognitive approach, the theory of Thinking Modes is used, which allows, by mean of its elements, to situate three ways of thinking the mathematical object: The Synthetic-Geometric mode, the Analytic-Arithmetic mode and the Analytic-Structural mode. From a historical-epistemological and mathematical study, the three thinking modes of the numeric system are characterized and two questionnaires about mathematical activities to five case studies are applied. The analysis of the questionnaires reveals a lack of articulation of the thinking modes, privileging the Analytic-Arithmetic mode and an absence of the transit towards the other two modes. This allows to conclude that there exists a fragmented understanding of the mathematical object and that it is necessary to increase the work from the geometric and structural point of view.
\end{abstract}

Keywords: complex numbers; number system; thinking modes; understanding numbers; articulators of thinking modes 


\section{INTRODUCCIÓN}

La enseñanza y aprendizaje del sistema de los números complejos (se hace referencia a sistema de los números complejos y no de conjunto de números complejos, porque es un conjunto dotado de estructura algebraica) ha sido una preocupación para la educación matemática desde hace algunos años. Investigaciones en distintas partes del mundo dan cuenta de diversas dificultades y errores en los procesos de apropiación de este objeto matemático, tanto a nivel universitario (Danenhower, 2000; Pardo y Gómez, 2007; Aznar et al., 2010; Distéfano et al., 2012; Ahmad y Shahrill, 2014; Smith et al., 2015) como escolar (Bagni, 2001; Panaoura et al., 2006; Martínez-Sierra y Antonio, 2009; Maumary y Maumary, 2015). Las evidencias apuntan, entre otras, a un fuerte trabajo desde lo aritmético-algebraico y uno casi nulo desde lo geométrico-vectorial (Danenhower, 2000; Panoura et al., 2006; Aznar et al., 2010; Distéfano et al., 2012). En lo específico, las investigaciones han reportado que los aprendices no tienen construido un significado geométrico de número complejo (Distéfano et al., 2012), reflejando una falta de flexibilidad en el uso de sus diferentes representaciones (Panoura et al., 2006; Aznar et al., 2012; Karakok et al., 2015; Smith et al., 2015) y, por tanto, una comprensión fragmentada de este. Y es que lograr la comprensión del sistema de los números complejos (SNC) no es una tarea fácil, pues dados los elementos que lo definen, requiere para su comprensión que los aprendices alcancen niveles superiores de abstracción y contrapongan ideas arraigadas sobre conceptos que han construido previamente en el sistema de los números reales.

En particular en Chile esta problemática no se percibe diferente. El estudio del SNC es abordado por los aprendices a nivel escolar, en tercer año de enseñanza media (16-17 años) y a nivel superior, en cursos iniciales de álgebra ( $\mathrm{y}$ en algunos cursos posteriores de universidad). Y aunque las investigaciones al respecto son muy escasas, un rápido análisis al currículo escolar nacional (Ministerio de Educación, 2009) permite observar que no hay referencia a técnicas que involucren aspectos geométricos de los números complejos, reflejando privilegiar un trabajo aritmético-algebraico. De hecho, indagando un poco más profundo en el texto escolar ministerial del nivel de tercer año medio (Saiz y Blumenthal, 2017), se aprecia que, aunque se presenta el enfoque geométrico del objeto número complejo, este no es más que el resultado de un trabajo aritméticoalgebraico previo; asimismo, no hay referencia a propiedades relevantes y distinciones con el sistema de los números reales o con la estructura de orden de los sistemas.

En este sentido, cabe preguntarse ¿cómo están comprendiendo el SNC aprendices del nivel escolar y universitario chileno? y ¿cómo es posible que dichos aprendices alcancen una comprensión profunda del objeto? Situados los antecedentes, por un lado en la falta de evidencias que permitan dar cuenta de la enseñanza y aprendizaje del SNC en el ámbito escolar y universitario chileno; y por otro, en el privilegio de un trabajo analítico del número complejo, dejando de lado características geométricas y estructurales que frenan la posibilidad de alcanzar niveles superiores de abstracción y enriquecer su aprendizaje; es apropiado indagar en el nivel de comprensión que tienen los aprendices sobre el objeto matemático, y en los elementos necesarios para alcanzar su comprensión profunda.

Desde la Didáctica de la Matemática la teoría de los Modos de Pensamiento nos brinda elementos teóricos para afrontar el estudio de la comprensión. El supuesto de investigación es que un estudiante alcanza una comprensión profunda del objeto cuando utiliza equilibradamente el pensamiento práctico y el pensamiento teórico, esto es, se comprende profundamente un tópico matemático cuando el aprendiz articula y transita por tres modos de pensamiento de ese fragmento de la matemática: Sintético-Geométrico, Analítico-Aritmético y Analítico-Estructural (Sierpinska, 2000).

La presente investigación se sitúa en aspectos cognitivos del aprendizaje del objeto —SNC— teniendo como propósito analizar cómo estudiantes de enseñanza media (16-18 años) y superior (licenciatura, pedagogía, magíster y doctorado, en matemática) comprenden este sistema numérico, y cómo es posible alcanzar su comprensión profunda. Con base en una aproximación epistemológica del objeto en estudio y desde un estudio de caso múltiple, se (1) definen los modos de pensar que sustentan su comprensión profunda; se (2) evidencian las formas de pensar que los aprendices del nivel escolar y universitario emplean y privilegian en situaciones matemáticas que involucran al objeto; y se (3) precisan tránsitos que logran y no logran dichos estudiantes entre las diferentes formas de pensar el SNC, dando cuenta de los elementos matemáticos que ponen en juego. Desde el desarrollo de estos objetivos de investigación se espera, entonces, contribuir a la discusión sobre la enseñanza y aprendizaje del SNC a nivel escolar y universitario, brindando un espacio para la reflexión sobre cómo producir aprendizajes significativos en los aprendices, en este caso desde la articulación de los modos de pensamiento involucrados en la apropiación del objeto matemático números complejos. 


\section{APROXIMACIÓN EPISTEMOLÓGICA DE LOS NÚMEROS COMPLEJOS}

En relación a la búsqueda de la comprensión profunda del SNC, surge la necesidad de describir su epistemología. Varios autores coinciden en la importancia y obligación de entender a los números complejos desde su evolución histórico-epistemológica, debido principalmente a que los aprendices reproducen en el aula varias de las dificultades y errores que afrontaron los matemáticos de su época en la construcción del número complejo (Bagni, 2001; Pardo y Gómez, 2007; Martínez-Sierra y Antonio, 2009). Más allá de contar en detalle cómo surgen los números complejos, es posible centrarse en el desarrollo de cuatro momentos cruciales o de conflictos cognitivos que fueron superados hacia el logro del desarrollo del SNC y que evidencian la presencia de diferentes enfoques.

\section{Momentos claves}

Según Artigue y Deledicq (1992) hay cuatro momentos cruciales en la epistemología de los números complejos: (1) la aparición de cantidades imaginarias en algoritmos operatorios; (2) el funcionamiento como herramienta y el encuentro con los ángulos y logaritmos; (3) las representaciones geométricas; y (4) la construcción algebraica. El primer momento guarda relación con primeras apariciones de las raíces cuadradas de cantidades negativas. Si bien desde el primer siglo después de cristo se pueden encontrar vestigios de la aparición de raíces cuadradas de números negativos, fue de la mano de matemáticos europeos en los siglos XVI y XVII que se empezó a discutir sobre ellas, al "extender la operación aritmética de la raíz cuadrada a números cualesquiera que apareciesen al resolver ecuaciones de segundo grado por el método de completar cuadrados" (Kline, 1972, p. 339). Las raíces cuadradas de cantidades negativas aparecieron en trabajos de algebristas italianos sobre técnicas de resolución de ecuaciones de tercer grado, que consistían en reducir la ecuación, con un cambio de variables, a una ecuación de segundo grado que se sabía resolver. Esta técnica, llamada técnica de Cardano, se utilizó en situaciones donde se obtenían soluciones reales para la ecuación de grado dos y luego se extendió a las ecuaciones que tenían como soluciones raíces cuadradas de números negativos.

El segundo momento, por su parte, vino con la aceptación de las cantidades imaginarias. Las raíces cuadradas de números negativos, no consideradas como números permanecieron por mucho tiempo como objetos sintácticos sin ningún significado. Sin embargo, ellas fueron más allá del contexto único de las ecuaciones a otros campos como la trigonometría, y comenzaron a verse como variables autónomas en expresiones funcionales y en los logaritmos. Por ejemplo, Leibniz y Bernoulli comenzaron a utilizar las cantidades imaginarias repetidamente en procesos de integración y cálculo de logaritmos, sin importar su presencia y sin entender su naturaleza. $Y$ hubo controversias al respecto que duraron unas cuatro décadas del siglo XVIII, que fueron resueltas por Euler desarrollando la base de una nueva teoría matemática para el tratamiento de logaritmos de números negativos e imaginarios. Euler introdujo la notación $i$ para $\sqrt{ }-1$ e intentó comprender lo que realmente eran las cantidades imaginarias; cometió algunos errores al operar con ellos, por ejemplo, escribe $\sqrt{ }_{-} \cdot \sqrt{ }-4=\sqrt{ }_{4}=2$ ya que $\sqrt{ } a \cdot \sqrt{ } b=\sqrt{ } a b$. A pesar de ello, Euler, encontró la manera de manipular varias fórmulas que incluían números imaginarios, suponiendo que se comportaban como números reales y dedujo la relación entre las funciones trigonométrica y exponencial, a través de la expresión $e^{i \theta}=$ $\cos (\theta)+i \operatorname{sen}(\theta)$. Así, las cantidades imaginarias se convirtieron en instrumentos necesarios para la formulación de resultados importantes del siglo XVIII, tal como el Teorema Fundamental del Álgebra, y comenzaron a presentar características de un objeto matemático, aunque permanecieron como simbólicos definidos, pero no construidos sin una interpretación real.

Seguidamente se produjo el tercer momento con el desarrollo de la representación geométrica. El desarrollo de la geometría de las cantidades imaginarias tuvo un alcance importante para cambiar el estatus de éstas a números y de propiciar luego su formalización como objetos matemáticos legítimos. Aunque hubo esfuerzos previos, la representación geométrica de los números complejos tiene sus primeras citas en los trabajos Wessel y Argand, entendiéndolos como líneas dirigidas. Ambos introdujeron el eje de los imaginarios asociando a $\sqrt{ }-1$ como una unidad perpendicular a 1 y mostraron cómo podían ser sumados y multiplicados geométricamente. Por ejemplo, Wessel argumentó que el producto debía tener dos propiedades: "la longitud del producto debía ser el producto de las longitudes de cada segmento y que el segmento producto debía diferir en la dirección de cada segmento factor por la misma cantidad angular que el otro segmento factor difería en dirección al compararlo con el segmento unidad" (Nahin, 1998, p.51). Más tarde Gauss, introduciendo el término de "número complejo" y usando $i=\sqrt{ }-1$, también los definió geométricamente, pero como puntos del plano y estableció una correspondencia uno a uno con los puntos del plano cartesiano, entendiendo que el número $a+b i$ estaría representado por el punto $(a, b)$ en el plano complejo. $\mathbf{w}$

Finalmente, el cuarto momento fue el de la construcción algebraica. Después que fue proporcionada la representación geométrica de los números complejos, los matemáticos se percataron de que éstos podían 
usarse para trabajar y representar vectores en el plano. Lo que los números complejos hacen para los vectores en el plano es proporcionar un álgebra para representarlos y operar con ellos. Hamilton realizó la primera definición algebraica rigurosa del SNC, entendiéndolos como pares ordenados de números reales. Hamilton señaló al respecto que un número complejo $a+b i$ no era "una suma en el sentido en que $2+3$ lo es" (Kline, 1972, p.1022); y añadió que el uso del signo + es un accidente histórico y bi no puede ser añadido a $a$. En este sentido, el número complejo $a+b i$ no es más que una pareja de pares ordenados $(a, b)$ de números reales. Al respecto Hamilton (1837) escribe: "en la teoría de los números, el símbolo $V_{-}-1$ es absurdo, y denota una extracción imposible, o un número meramente imaginario; pero en la teoría de los pares, el mismo símbolo $V_{-1}$ es significativo, y denota una extracción posible o un par real, a saber (...) la raíz cuadrada principal del $\operatorname{par}(-1,0)$. Por lo tanto (...) para cualquier par $\left(a_{1}, a_{2}\right) ;\left(a_{1}, a_{2}\right)=a_{1}+a_{2} \sqrt{ }-1$ " (p.107, traducido). Con esta visión, el SNC no sólo estuvo fundamentado lógicamente sobre la base de los números reales, sino que también "el misterioso $\sqrt{ }-1_{-1}$ era evitado por completo" (Kline, 1972, p.1022).

En definitiva, es posible establecer un isomorfismo $\mathrm{R} \times \mathrm{R} \rightarrow \mathrm{C}$, que asocia cada elemento $(a, 0)$ con el número real $a$ y a cada $(0, b)$ con $b i$, donde $i$ corresponde al par ordenado $(0,1)$. En general considerando estas notaciones se tiene que $(a, b)=(a, 0)+(b, 0)=a+b i$. Lo que permite escribir: $\mathrm{C}=\mathrm{R}^{2}=\{(a, b): a, b \in \mathrm{R}\}$ $=\{a+b i: a, b \in \mathrm{R}\}$.

\section{La aproximación epistemológica}

Los momentos epistemológicos descritos permiten sustentar la existencia de diferentes formas de pensar el SNC, durante su construcción matemática. De este modo, y de acuerdo con Martínez-Sierra y Antonio (2009) y Pardo y Gómez (2007) es posible identificar cuatro etapas en el desarrollo de la conceptualización del objeto (Fig. 1). Cada etapa pone de relieve distintas formas de pensamiento: hay presencia del pensamiento algebraico y analítico en los dos primeros momentos epistemológicos, al ligar las incipientes apariciones de las raíces cuadradas de cantidades negativas y a la aceptación del uso de dichas cantidades; y también emerge el pensamiento geométrico y formal, con base en el tercer y cuarto momento, al relacionar la introducción del eje de los imaginarios asociando a $V_{-1}$ como una unidad perpendicular a 1 y a su consideración como vectores y pares ordenados en el plano complejo.

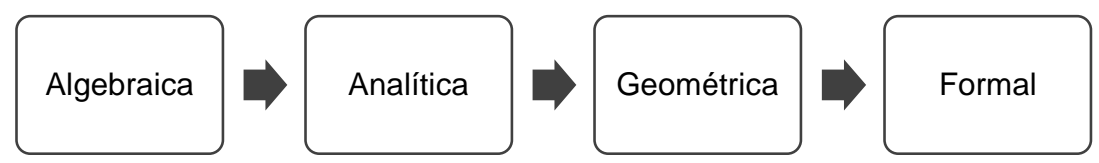

Fig. 1: Etapas en la construcción del número complejo.

También, en lo particular es posible identificar en al análisis histórico epistemológico elementos de la matemática que permitieron posicionar a los matemáticos en un tipo de pensamiento y movilizarlos hacia otros, esto es: (a) aceptar las cantidades imaginarias como raíces de una ecuación cúbica (pensamiento algebraico de las cantidades imaginarias); (b) el uso de las cantidades imaginarias como variables en logaritmos e integrales (pensamiento analítico); (c) la representación de las cantidades imaginarias como puntos y segmentos de línea dirigida en el plano dando inicio a su formalización desde su forma de pares ordenados $(a, b)$ (pensamiento geométrico); y (d) la idea de multiplicar módulos y sumar argumentos como idea que justifica la perpendicularidad del eje imaginario (pensamiento geométrico). Todos estos aspectos, destacan distintas formas de pensar el SNC en el transcurso de su conceptualización. Esto conlleva a considerar, la búsqueda de la comprensión profunda del objeto número complejo desde los modos de pensamiento, como marco teórico.

\section{LOS MODOS DE PENSAMIENTO}

Los modos de pensamiento (Sierpinska, 2000) constituyen un marco teórico cognitivo que se caracteriza por proporcionar elementos que interpretan la comprensión de un objeto matemático por parte de un aprendiz. Fue desarrollado al estudiar algunos aspectos del razonamiento de estudiantes universitarios en el aprendizaje del Álgebra Lineal (AL); en particular, se observó que los estudiantes comprendían el AL en muchos aspectos de la teoría con un enfoque más práctico que teórico, afectando negativamente el razonamiento en el ámbito del álgebra (Parraguez, 2012). Así, la teoría se desarrolla dando luces acerca de la conformación que puede tener el pensamiento durante los aprendizajes de la matemática. El pensamiento práctico y el pensamiento teórico emergen para instaurar una dualidad que no tiene por qué convertirse en polos opuestos. De hecho, se espera todo lo contrario - la articulación entre ambos tipos de pensamientolo que permitirá una mejor interpretación de la comprensión cabal del objeto de estudio en los aprendices. 
Sierpinska, Nnadozie y Oktaç (2002) distinguen el pensamiento práctico del pensamiento teórico: una forma práctica de pensar está dirigida a una acción inmediata, y una forma de pensamiento teórico a la reflexión referida a conexiones conceptuales, esto es, el pensar por el bien de pensar, reflexionar sobre posibles resultados de una acción, no para llevar a cabo la acción. Así, se asocia el pensamiento práctico a una forma de pensar geométrica (de la geometría sintética) y el pensamiento teórico a una forma de pensar analítica. En la superación del obstáculo epistemológico producido al confrontar el pensamiento práctico con el pensamiento teórico, Sierpinska (2000) identifica la existencia de tres categorías de pensamiento relacionadas con el uso de los lenguajes gráfico, algebraico y abstracto del AL: Sintético-Geométrico (SG), Analítico-Aritmético (AA) y Analítico-Estructural (AE). La autora precisa que estas categorías, llamadas modos de pensamiento, surgieron históricamente de forma secuencial y que la aparición de cada una de ellas no eliminó a los otras dos; además, señala que son igualmente útiles en especial cuando están en interacción (Fig. 2).

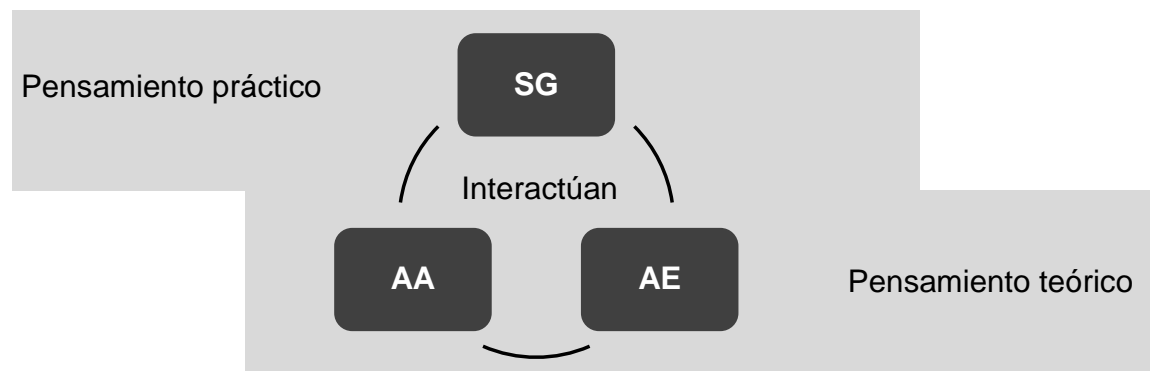

Fig. 2: Modos de pensar de Sierpinska en el contexto del pensamiento práctico y teórico.

Los modos de pensamiento son formas de ver y entender los objetos matemáticos, esto depende de los tipos de relaciones y objetos que evoquemos al momento de pensar en un objeto o al intentar resolver una tarea; ya sea porque se le solicita al aprendiz razonar en un determinado registro de representación o porque la tarea misma así lo requiere. De esta forma, el modo SG usa el lenguaje geométrico teniendo visualmente puntos, líneas, planos, figuras y cuerpos geométricos; la visualización matemática, en el sentido de Zimmermann y Cunningham (1991), juega un rol fundamental en lo que es la resolución de problemas en este modo de pensamiento. Por su parte, en el modo AA los objetos se representan mediante relaciones numéricas o fórmulas; por ejemplo, el vector pasa a ser un par ordenado, las rectas ecuaciones lineales; en este modo, el pensamiento es teórico desde el momento en que el estudiante debe interpretar los objetos a partir de ciertas relaciones numéricas o simbólicas. En el modo de pensar AE se recurre a las propiedades de los objetos o a su caracterización a través de axiomas, esto es, los objetos son representados por las propiedades que poseen o los axiomas que permiten explicarlos; por ejemplo, las matrices, las funciones que pueden ser vistas como elementos generadores de un espacio vectorial, etc.

La principal diferencia entre los modos sintético y analítico, afirma la autora, es que en el modo sintético los objetos son aproximados directamente a la mente, la cual trata de describirlos, es decir, de manera natural, mientras que en el modo analítico, estos objetos se aproximan de forma indirecta, de hecho son construidos solamente por la definición de las propiedades de sus elementos; por su parte la diferencia entre los modos, analítico aritmético y estructural, es que en el primero la interpretación del objeto es reflejada por una fórmula que permite calcularlo, mientras que en el segundo, es por un grupo de determinadas propiedades.

Así, la teoría caracteriza estos tres modos de pensamiento esperando una articulación entre ellos, lo que permitirá la comprensión profunda de objetos matemáticos por parte de los aprendices. Dicho de otro modo, para esta teoría comprender un objeto matemático es poder abordarlo articuladamente desde SG, AA y AE, alcanzando niveles superiores de abstracción. Más aún, los modos de pensamiento no sólo son formas de pensar y entender los objetos matemáticos, sino que también actúan como herramientas heurísticas al resolver problemas (Parraguez, 2012). SG, AA y AE constituyen una vía de acceso a los objetos matemáticos, aunque la coordinación y el tránsito entre ellos permiten por un lado un pensamiento más versátil, y por otro, mostrar diferentes facetas del objeto matemático, ofreciendo diferentes aspectos según el registro en el que se ubique. A pesar de que este marco nace para dar respuestas a problemáticas del AL, con esta investigación se pretende seguir extendiendo este referente teórico a otros tópicos matemáticos, tal como Pinto y Parraguez (2017) quienes abordan el estudio de la comprensión de la derivada desde su perspectiva local.

\section{Modos de pensar el sistema de los números complejos}

Abordar la comprensión del SNC desde los modos de pensamiento es posible, pues la aproximación epistemológica del número complejo sustenta la existencia de diferentes formas de pensarlo a lo largo de su conceptualización (Fig. 1). De acuerdo a ello, se han caracterizado tres modos de pensar el SNC (Fig. 3). 

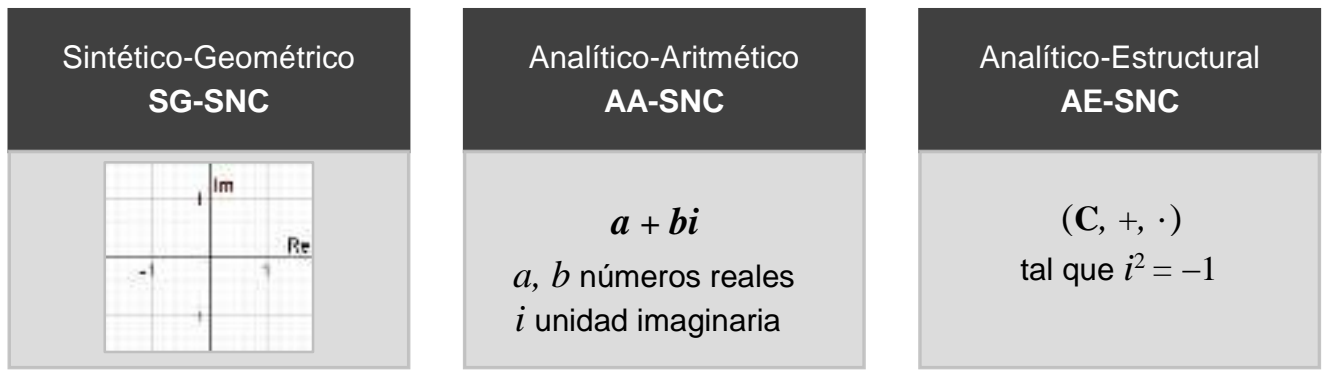

Fig. 3: Caracterización de los modos de pensamiento para el SNC.

En el modo de pensar SG, el SNC se muestra a través de su representación como el plano complejo; se consideran en este modo los puntos y vectores del plano y todo lo que es posible realizar a partir de él, como las construcciones con regla y compás de las operaciones suma y producto. El modo AA del objeto se expresa al representar y operar con la expresión algebraica $a+b i$ con $a, b$ números reales e $i$ la unidad imaginaria; aquí se considera la definición de fórmulas para encontrar las operaciones, el módulo, etc. Si el estudiante sólo se sitúa en este modo de pensar, se dirá que él entiende a los números complejos como un conjunto numérico y no como un sistema. $\mathrm{Y}$ el modo $\mathrm{AE}$, por su parte, se revela desde su representación como estructura de cuerpo tal que $i^{2}=-1$; este último modo considera la estructura algebraica y de orden del SNC, sus propiedades y axiomas. Un ejemplo que da cuenta de estos elementos es el teorema de De Moivre (Carreño y Cruz, 2011) y el Teorema Fundamental del Álgebra (Hungerford, 2003).

La Tabla 1, que se presenta a continuación, sintetiza los elementos matemáticos que se han considerado a priori - como argumentos observables - para evidenciar el trabajo de los estudiantes participantes de la investigación en los distintos modos de pensar el SNC.

Tabla 1: Elementos matemáticos observables en los modos de pensar el SNC.

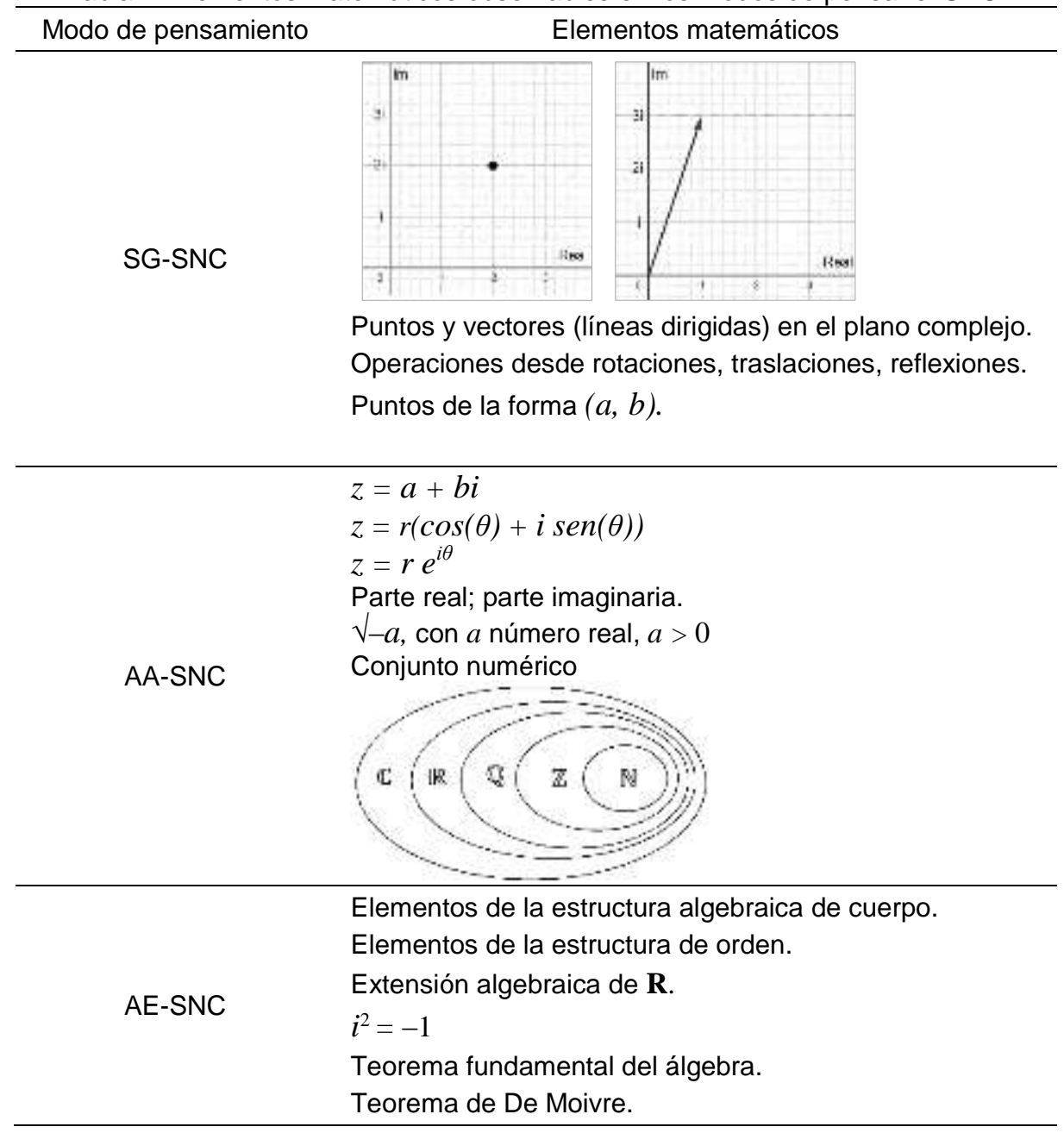


Considerar al SNC como una expresión algebraica en el contexto en que surge, es relevante para aceptar su existencia como objeto matemático (hecho que se interpreta como modo de pensar AA-SNC), tal como los algebristas en la resolución de las ecuaciones cúbicas. Pero también se considera importante entender a los números complejos como un sistema con estructura de cuerpo, donde se cumplen propiedades y axiomas que nos permiten entenderlos y trabajar con ellos, tal como $i^{2}=-1$ (interpretado como modo AE-SNC). Además, considerar al sistema en su carácter geométrico, vale decir, como el plano complejo, es relevante para poder transitar entre los modos AA-SNC y AE-SNC, y para comprender sus propiedades e incluso sus operaciones. Tal como se ha expuesto, el marco teórico brinda elementos para interpretar, describir y escribir el objeto en estudio mediante la definición de los tres modos de pensamiento. Se espera entonces, que los aprendices logren transitar por ellos para llegar a la comprensión profunda del objeto.

\section{METODOLOGÍA}

Este estudio se enmarca en una metodología de tipo cualitativa con una postura epistemológica hermenéutica interpretativa, centrada en cómo se comprende el objeto matemático SNC; es decir, se busca la comprensión de un fenómeno profundo donde los datos son las palabras de las personas y al mismo tiempo la matemática que se pone en juego en una situación matemática específica.

\section{Etapas de la investigación}

El estudio se ha llevado a cabo en dos etapas (Tabla 2). En relación al primer objetivo de investigación se realizó un estudio epistemológico y matemático del SNC; la técnica utilizada fue la revisión bibliográfica; con este análisis se caracterizaron los modos de pensar SG-SNC, AA-SNC y AE-SNC. Y, de acuerdo con el segundo y tercer objetivo de investigación y desde un estudio de caso múltiple, se realizó la segunda etapa, que correspondió a la aplicación de dos cuestionarios de preguntas abiertas y actividades matemáticas.

Tabla 2: Etapas de la investigación.

\begin{tabular}{lll}
\hline \multicolumn{2}{l}{ Etapa 1: estudio epistemológico y matemático del objeto } \\
\hline Diseño metodológico & $\begin{array}{l}\text { Antecedentes epistemológicos para la caracterización } \\
\text { de los modos de pensar el SNC. }\end{array}$ \\
\hline Revisión bibliográfica & Instrumento & Población \\
\hline Etapa 2: diseño y aplicación de preguntas y actividades matemáticas & Caso I \\
\hline Diseño metodológico & Cuestionario A & Caso II \\
\cline { 2 - 3 } Estudio de caso múltiple & Cuestionario B & Caso III \\
\cline { 2 - 3 }
\end{tabular}

\section{El estudio de caso como método de investigación}

Desde lo cualitativo se ha seleccionado el estudio de caso como método para alcanzar los objetivos propuestos. Éste corresponde "al estudio de la particularidad y de la complejidad de un caso singular, para llegar a comprender su actividad en circunstancias importantes" (Stake, 2010, p.11). Su propósito fundamental es comprender en profundidad el caso que interesa "tanto por lo que tiene de único como por lo que tienen de común" (Stake, 2010, p.15). Se trata entonces, de un estudio procesual, sistemático y lo más detallado posible. Específicamente esta investigación utiliza el estudio de caso múltiple, que define varios casos a la vez para estudiar y describir la realidad, preocupándose de seleccionar aquellos que ofrezcan mejores y mayores oportunidades de aprendizaje del problema de estudio.

\section{Los casos de estudio}

Cada caso de estudio persigue un propósito, por lo que la elección de los mismos no es según criterios estadísticos sino por razones teóricas, se entiende que "la investigación con estudio de casos no es una investigación de muestras" (Stake, 2010, p.17) que pretenda establecer leyes generales, más bien se pretende comprender realidades concretas dentro de un contexto global. Recordando que esta investigación pretende evidenciar los modos de pensamiento del sistema números complejos que privilegian los estudiantes y la articulación que hay entre ellos para la comprensión profunda del objeto matemático en cuestión, se consideraron los siguientes criterios para la conformación de los casos: (a) nivel de conocimientos de los estudiantes sobre el objeto matemático SNC; (b) formación matemática de los participantes; (c) accesibilidad de las investigadoras. 
Así, se consideraron estudiantes destacados en matemáticas: (a) estudiantes de enseñanza media con promedio de notas superior a 6,5 (en una escala de uno a siete) en la asignatura de matemáticas; (b) estudiantes universitarios con un promedio superior a 5,0 (en una escala de uno a siete) y con sus ramos de la malla académica al día. Los estudiantes de educación superior pertenecen a la Facultad de Ciencias de la Universidad de Chile; se consideraron de esta casa de estudios dado los contactos de una de las investigadoras con docentes de esta facultad que ayudaron con el acceso a los estudiantes; por su parte, la llegada a los estudiantes de enseñanza media fue por colegas profesores que hicieron las gestiones en sus establecimientos para permitir el ingreso de las investigadoras a aplicar el instrumento; estos corresponden a estudiantes de cuatro colegios de Santiago en las comunas de La Florida, Puente alto, Nuñoa y Providencia. Así, los casos quedaron constituidos tal como se presenta en la Tabla 3.

Tabla 3: Participantes y casos de estudio.

\begin{tabular}{|c|c|c|c|c|}
\hline Casos & Participantes & Nivel & Características & Identificación \\
\hline Caso I & $\begin{array}{l}5 \text { estudiantes de } 3^{\circ} \\
\text { de enseñanza media }\end{array}$ & Escolar & $\begin{array}{l}\text { Han estudiado el objeto por } \\
\text { primera vez. }\end{array}$ & $\begin{array}{l}\text { E1; E2; E3 E4; } \\
\text { E5 }\end{array}$ \\
\hline Caso II & $\begin{array}{l}6 \text { estudiantes de } 4^{\circ} \\
\text { de enseñanza media. }\end{array}$ & Escolar & $\begin{array}{l}\text { Han estudiado el objeto por } \\
\text { primera o segunda vez. }\end{array}$ & $\begin{array}{l}\text { E6; E7; E8 E9; } \\
\text { E10; E11 }\end{array}$ \\
\hline Caso III & $\begin{array}{l}4 \text { estudiantes de } 4^{\circ} \\
\text { año de pedagogía en } \\
\text { matemática y física. }\end{array}$ & $\begin{array}{l}\text { Universitario } \\
\text { pregrado }\end{array}$ & $\begin{array}{l}\text { Han abordado el objeto en los } \\
\text { cursos de álgebra y sistemas } \\
\text { numéricos. }\end{array}$ & $\begin{array}{l}\text { E12; E13 E14; } \\
\text { E15 }\end{array}$ \\
\hline Caso IV & $\begin{array}{l}2 \text { estudiantes de } 4^{\circ} \\
\text { año licenciatura en } \\
\text { matemática. }\end{array}$ & $\begin{array}{l}\text { Universitario } \\
\text { pregrado }\end{array}$ & $\begin{array}{l}\text { Han abordado el objeto en los } \\
\text { cursos de álgebra, sistemas } \\
\text { numéricos y variable compleja. }\end{array}$ & E16; E17 \\
\hline Caso V & $\begin{array}{l}2 \text { estudiantes de } \\
\text { magister y } 1 \\
\text { estudiante de } \\
\text { doctorado, en } \\
\text { matemática. }\end{array}$ & $\begin{array}{l}\text { Universitario } \\
\text { postgrado }\end{array}$ & $\begin{array}{l}\text { Han estudiado el objeto en cursos } \\
\text { de álgebra, sistemas numéricos, } \\
\text { análisis y variable compleja. }\end{array}$ & E18; E19; E20 \\
\hline
\end{tabular}

\section{Recogida de datos}

Los estudios de caso utilizan técnicas cualitativas de recolección de datos, como la observación, la entrevista, la revisión de documentos, cuestionarios, entre otros. En esta investigación se utiliza como instrumento de recogida de datos dos cuestionarios escritos, constituidos por preguntas abiertas (Tabla 4) y actividades matemáticas (Tabla 5).

Tabla 4: Preguntas de los cuestionarios.

\begin{tabular}{|c|c|c|c|}
\hline Pregunta & Cuestionario & Pregunta & $\begin{array}{l}\text { Modos de pensar } \\
\text { a observar }\end{array}$ \\
\hline P1 & $\mathrm{CA}-\mathrm{CB}$ & $\begin{array}{l}\text { ¿Qué es lo que primero viene a tu mente cuando escuchas "números } \\
\text { complejos"? }\end{array}$ & $S G / A A / A E$ \\
\hline $\mathrm{P} 2$ & $\mathrm{CA}-\mathrm{CB}$ & $\begin{array}{l}\text { ¿Cómo explicarías a un(a) compañero(a) qué son los números } \\
\text { complejos? Escribe cada paso de lo que harías o dirías. }\end{array}$ & $S G / A A / A E$ \\
\hline P3 & CB & $\begin{array}{l}\text { ¿Qué crees que distingue a este sistema numérico de otros } \\
\text { sistemas numéricos? Explica. }\end{array}$ & $S G / A A / A E$ \\
\hline P4 & CB & ¿Es el SNC un cuerpo conmutativo? ¿Por qué? Justifica. & $A E$ \\
\hline P5 & CB & ¿Se puede ordenar el SNC? ¿Por qué? Justifica tu respuesta. & $A E$ \\
\hline
\end{tabular}

La necesidad de hacer dos cuestionarios diferentes radicó en los distintos niveles de conocimiento matemático que poseen los informantes; por ello, se aplicó un cuestionario A (CA), a los informantes del nivel escolar (Caso I y II), y un cuestionario B (CB) a los informantes del nivel universitario (Casos III, IV y V). EI CA constó de dos preguntas abiertas (P1-P2) y nueve actividades matemáticas (A1-A9), mientras que el CB tuvo las mismas preguntas y actividades, más tres preguntas abiertas (P3-P5) y una actividad matemática adicional (A10). El propósito de añadir ítems al cuestionario $B$, fue para evidenciar mejor los elementos matemáticos que articulan los modos de pensamiento SG-SNC, AA-SNC y AE-SNC. 
Tabla 5: Actividades de los cuestionarios.

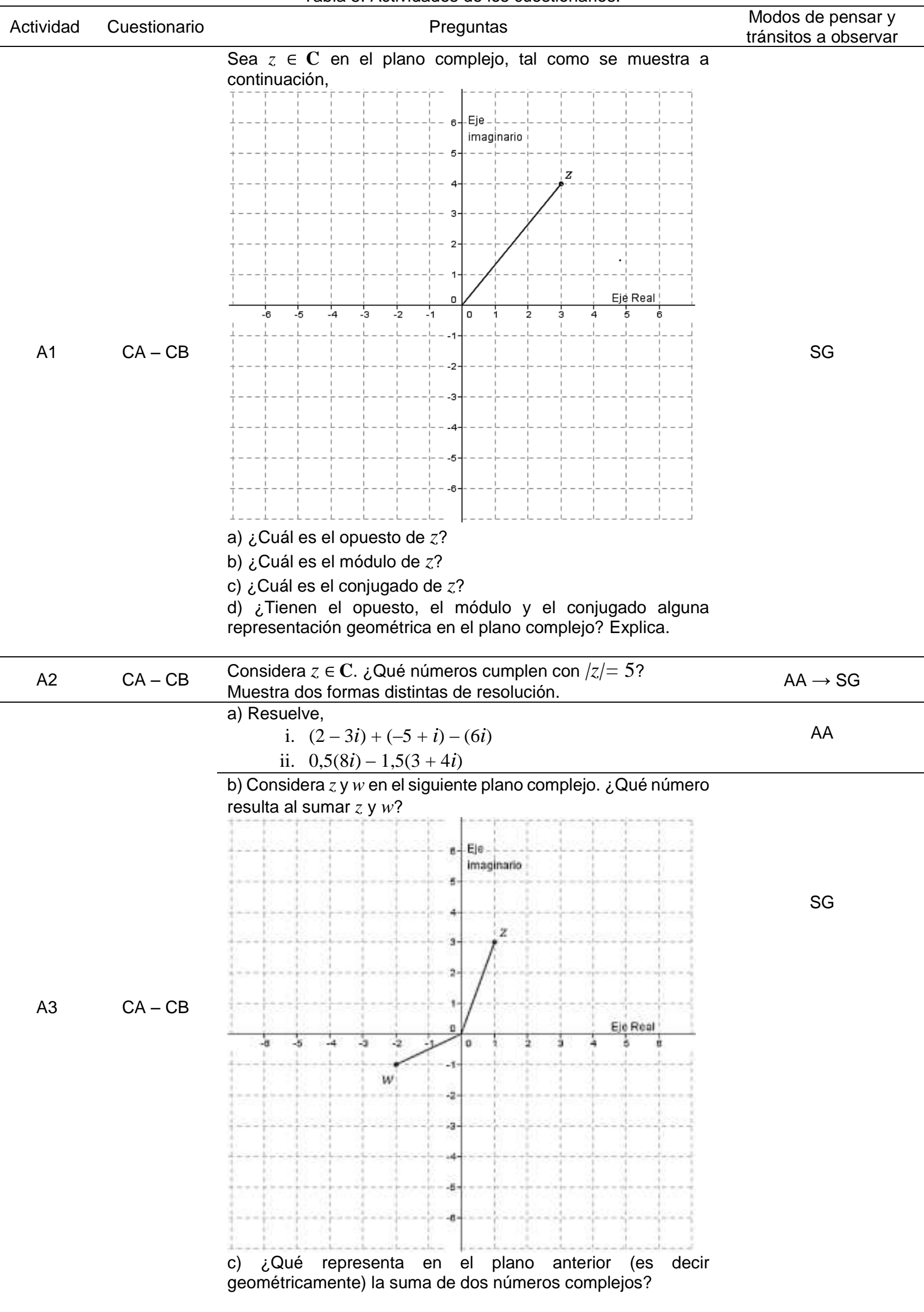


Tabla 5: (continuación).

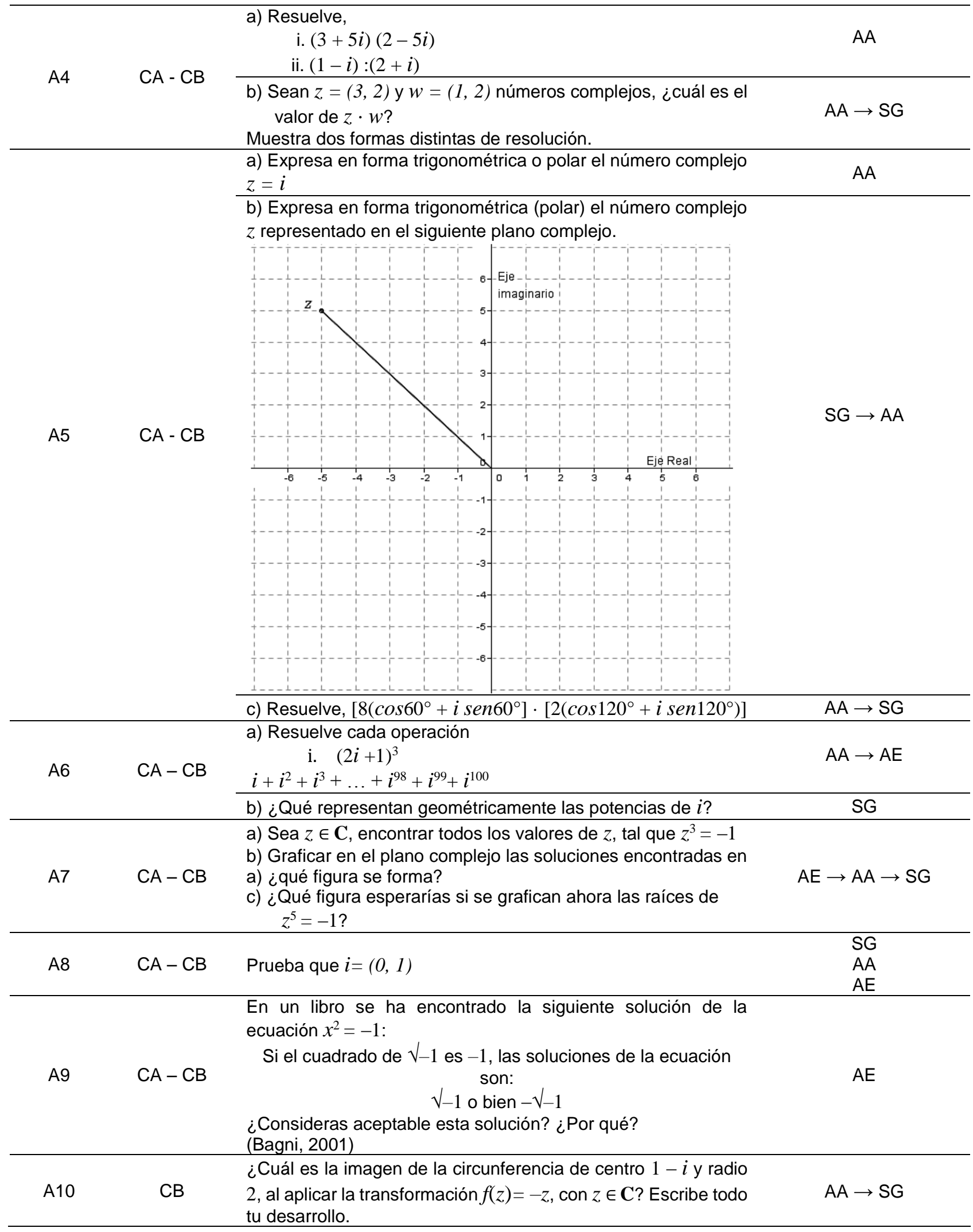

La validación de los cuestionarios se hizo a través de la consulta a expertos, un tipo de validación del contenido del instrumento en el cual un panel de jueces o expertos en la disciplina analizan si el contenido de las preguntas y actividades planteadas se refieren a lo que se pretende evidenciar (Creswell, 2012). El panel de expertos fue constituido por una doctora y un magíster en didáctica de la matemática, dos magísteres en matemáticas y dos profesores de matemática de enseñanza media, todos pertenecientes al Grupo Cognitivo del Instituto de Matemáticas de la Pontificia Universidad Católica de Valparaíso. Los cuestionarios fueron 
aplicados por las investigadoras a los cinco casos de estudio. A cada informante se le proporcionó una copia del cuestionario en la que cada pregunta y actividad ocupó una hoja. Los informantes tuvieron alrededor de dos horas para contestar y sólo se respondieron dudas acerca de la comprensión de los enunciados. Una vez aplicados, éstos fueron digitalizados para proceder a su análisis.

\section{Análisis}

Se realizó un análisis a priori de las preguntas y actividades de los cuestionarios $C A$ y $C B$, explicitando posibles respuestas de los participantes; $y$ luego, se realizó un análisis a posteriori basado en la interpretación directa y la suma categórica (Stake, 2010). Esto quiere decir que se hizo una interpretación directa de cada respuesta individual y a partir de estas se observaron relaciones en y entre cada caso de estudio. Esto se desarrolló bajo una profunda discusión de las investigadoras, llegando a consensuar las diferencias. En particular, el análisis a posteriori de cada pregunta y actividad se llevó a cabo considerando los siguientes criterios: (1) los Modos de Pensamiento que priorizan los informantes al enfrentarse a las preguntas y actividades planteadas en los distintos modos de comprender el SNC; (2) los tránsitos que logran y no logran los informantes entre los modos de comprender el SNC; (3) los elementos de la matemática que los informantes ponen en juego al desarrollar los cuestionarios; y (4) las dificultades y/o errores que se presentaron en el desarrollo de las preguntas y actividades.

\section{ANÁLISIS DE LOS CUESTIONARIOS}

Los cuestionarios fueron diseñados en base a los modos de pensamiento del SNC (Fig. 3). Para poder evidenciar y explorar cómo es la articulación de los modos SG-SNC, AA-SNC y AE-SNC y el privilegio que le dan los informantes a cada uno de ellos, las preguntas y actividades del cuestionario estuvieron situadas en un modo de pensar, e invitaron en algunos casos a transitar hacia los otros modos. De esta manera se esperó, por medio de la respuesta del informante, dar cuenta del modo en el que se sitúa y las conexiones que establece entre los distintos modos, mediante los elementos matemáticos que pone de manifiesto.

En primer lugar, las preguntas P1, P2, P3, P4 y P5 permiten dar cuenta de las distintas definiciones que tienen los informantes acerca del SNC. Cabe señalar que las respuestas reúnen elementos del pensamiento práctico y del pensamiento teórico del informante, según haga referencia a cuestiones algebraicas, analíticas, geométricas o estructurales del objeto. En particular P1 y P2 apuntan a evidenciar qué modo de pensar, SGSNC, AA-SNC o AE-SNC, privilegian los estudiantes de enseñanza media y superior, al referirse al concepto de número complejo, poniendo atención a los elementos matemáticos que muestran al articular dos o más modos de pensar en sus respuestas. Por su parte, las preguntas P3, P4 y P5 guardan relación con evidenciar propiedades características del SNC como su estructura algebraica y estructura de orden. En este sentido, estas preguntas permitieron indagar específicamente en el modo de pensar AE-SNC.

En segundo lugar, las actividades matemáticas permitieron indagar en el conocimiento de los participantes sobre cada modo de pensamiento y sobre la articulación o tránsito entre ellos. La A1 tiene como objetivo, situar al estudiante en el modo de pensar SG, evidenciar si éste lo privilegia para responder o recurre al modo AA-SNC de pensar; además de explorar si el opuesto, el módulo y el conjugado permiten movilizar a los estudiantes al modo SG-SNC del SNC.

Las actividades A2, A3, A4, A5 y A10, por su parte, se centran en los modos SG-SNC, AA-SNC y su articulación. La A2, indaga en el valor absoluto de un número complejo como un articulador entre los modos; además permite mirar qué modo de pensar privilegian si SG-SNC o AA-SNC (o ambos) para responder, cuando están situados en AA-SNC. La A3, por su lado, tiene por objetivo indagar el tránsito entre SG-SNC hacia AA-SNC a través de la suma de números complejos; primero en a) se sitúa al estudiante en el modo AA-SNC y luego en las preguntas b) y c) se sitúa en el modo de pensar SG-SNC; se pretendió analizar si los estudiantes al sumar números complejos privilegian el modo SG-SNC o AA-SNC para responder y si conocen la suma geométrica en el plano complejo. Por otra parte, la A4 permite evidenciar si el número complejo como par ordenado permite el tránsito de AA-SNC a SG-SNC; además, analizar si los estudiantes recurren a la forma trigonométrica (más cerca de SG-SNC) o binómica (AA-SNC) para realizar la multiplicación de los números. Del mismo modo, la A5 tiene como propósito indagar en el tránsito entre SG-SNC a AA-SNC, pero mediante las coordenadas trigonométricas, situando al estudiante en el modo AA-SNC y luego en b) en el modo SG-SNC; además con la pregunta c), se analiza si los estudiantes conocen las cualidades de multiplicar en la forma trigonométrica dos números complejos (suma los argumentos y multiplica los módulos). Por último, la actividad A10, se presenta para evidenciar el rol de las transformaciones, en este caso $f(z)=-z$, en el tránsito de AA-SNC a SG-SNC. 
Desde otra perspectiva, la actividad, A6, apunta a indagar por un lado si los estudiantes privilegian el modo de pensar AA-SNC para resolver las operaciones y si recurren al Teorema de De Moivre para calcular el caso de la potencia a) i., coordinando AA-SNC con AE-SNC. Además, se pretende evidenciar con la pregunta b) la conexión con el modo de pensar SG-SNC que puedan establecer los estudiantes al representar las potencias de $i$ en el plano complejo. De la misma manera, el propósito de la A7 es explorar si los estudiantes al calcular las raíces cúbicas de -1 transitan por AE-SNC, AA-SNC y SG-SNC. Sobre todo, mirar la conexión que podrían establecer entre AE-SNC y AA-SNC.

En otro orden, la A8 se fundamenta en la necesidad de indagar en el modo de pensar que privilegiaran los estudiantes para explicar que $i=(0,1)$. Se espera que recurran al modo de pensar AE-SNC más que a los otros dos modos, justificándolo desde la construcción como pares ordenados que se hace del sistema numérico de los números complejos. Finalmente, la A9, extraída de Bagni (2001), permite evidenciar si los estudiantes, a pesar de haber trabajado todo el cuestionario, aceptan o no la existencia del SNC o si privilegian los modos de pensar el sistema de los números reales.

\section{RESULTADOS}

Los resultados se derivan de un análisis a posteriori de las preguntas y actividades de los cuestionarios para cada caso de estudio. Dicho análisis tomó como referencia los procedimientos, estrategias y argumentos observables presentados por los estudiantes en el desarrollo de los cuestionarios.

Hallazgos para los Casos I y II: estudiantes de educación escolar

1. Privilegio por el modo AA-SNC: los informantes, en su mayoría, se sitúan y priorizan el modo de pensar AA-SNC para responder a las preguntas y actividades, aunque éstas estén presentadas desde SG-SNC o AE-SNC. Esto los lleva a entender a los números complejos como expresiones algebraicas, replicando errores del álgebra (con los signos, los paréntesis, etc.), y a usar relaciones mecánicamente como fórmulas sin sentido. Las Fig. 4, por ejemplo, muestra la respuesta de E3 a la P2 desde el modo de pensar AA-SNC, considerando al SNC como un conjunto de números que se puede operar mediante fórmulas. En las actividades, también es posible notar el privilegio por AA-SNC. En la Fig. 5 se muestra como el informante E8 se sitúa en AA-SNC y sin realizar tránsitos a otros modos, no logra dar respuesta correcta a la actividad. Además, considera la notación $i=\sqrt{ }-1$ que dificulta el desarrollo del problema.

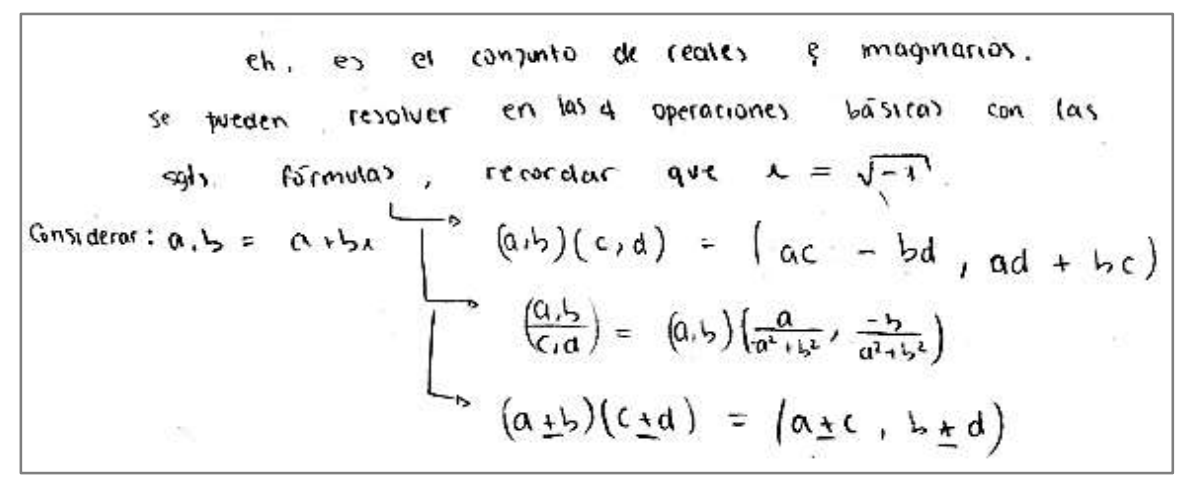

Fig. 4: Respuesta E3 a P2.

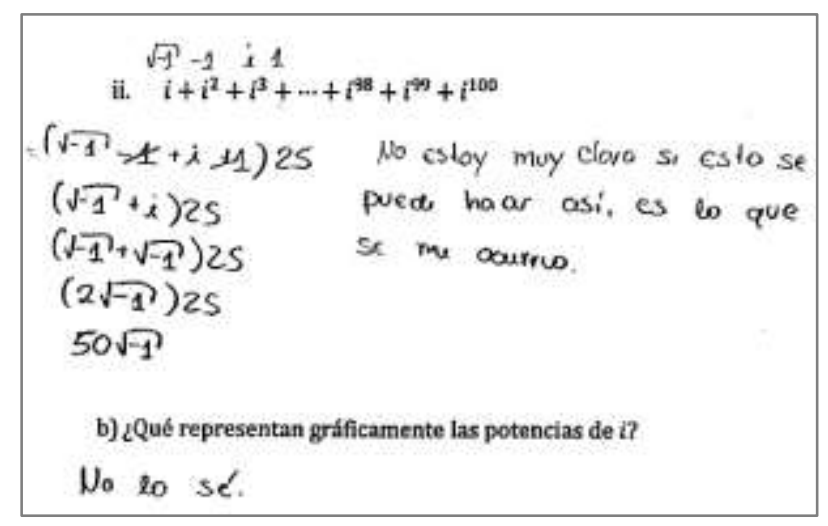

Fig. 5: Respuesta de E8 a A6. 
2. Hay tránsito $A A-S N C \rightarrow S G-S N C$, pero no se alcanza SG-SNC: Los estudiantes de educación escolar no se sitúan en SG-SNC para responder a las actividades, aunque den cuenta de algunos aspectos geométricos del sistema numérico. Por ejemplo, E7 en la A1, señala que el módulo de un número complejo es la distancia de $z$ al origen del plano complejo, sin embargo, resuelve con $\sqrt{ }\left(x^{2}+y^{2}\right)$. A pesar de privilegiar un pensamiento AA-SNC, algunos informantes evidencian el tránsito AA-SNC $\rightarrow$ SG-SNC, lo que les permite desarrollar con éxito la actividad. Por ejemplo, en la A2 quienes dan cuenta de la conexión entre AA-SNC y SG-SNC resuelven correctamente (Fig. 6), mientras que quienes se mantienen en el modo AA-SNC solo listan casos particulares.

En el desarrollo de la $\mathrm{A} 3$, que considera la suma de dos números complejos en el plano complejo, también es posible evidenciar que los informantes en su mayoría no se sitúan en SG-SNC para responder. Dos estudiantes exhibieron estrategias en el plano complejo, mientras que el resto realizó la suma algebraica desde las notaciones $a+b i$ o $(a, b)$. Los informantes que utilizaron argumentos geométricos para realizar la suma lo hicieron desde el número complejo como vector, el que permite realizar movimientos en el plano como los de traslación y rotación. La Tabla 5 muestra el desarrollo del informante E2 desde SG-SNC y el de E4 desde AA-SNC.

Por su parte, para la multiplicación de dos números complejos, ninguno de los informantes fue capaz de hacerla desde el modo de pensar SG-SNC e incluso las actividades A5 y A7 no pudieron ser resueltas debido a que la notación trigonométrica o polar de los números complejos no les fue enseñada. En este sentido, los estudiantes de estos casos de estudio no alcanzan el modo SG-SNC, al carecer de elementos y argumentos geométricos que les permitan resolver las actividades desde y hacia SG-SNC.

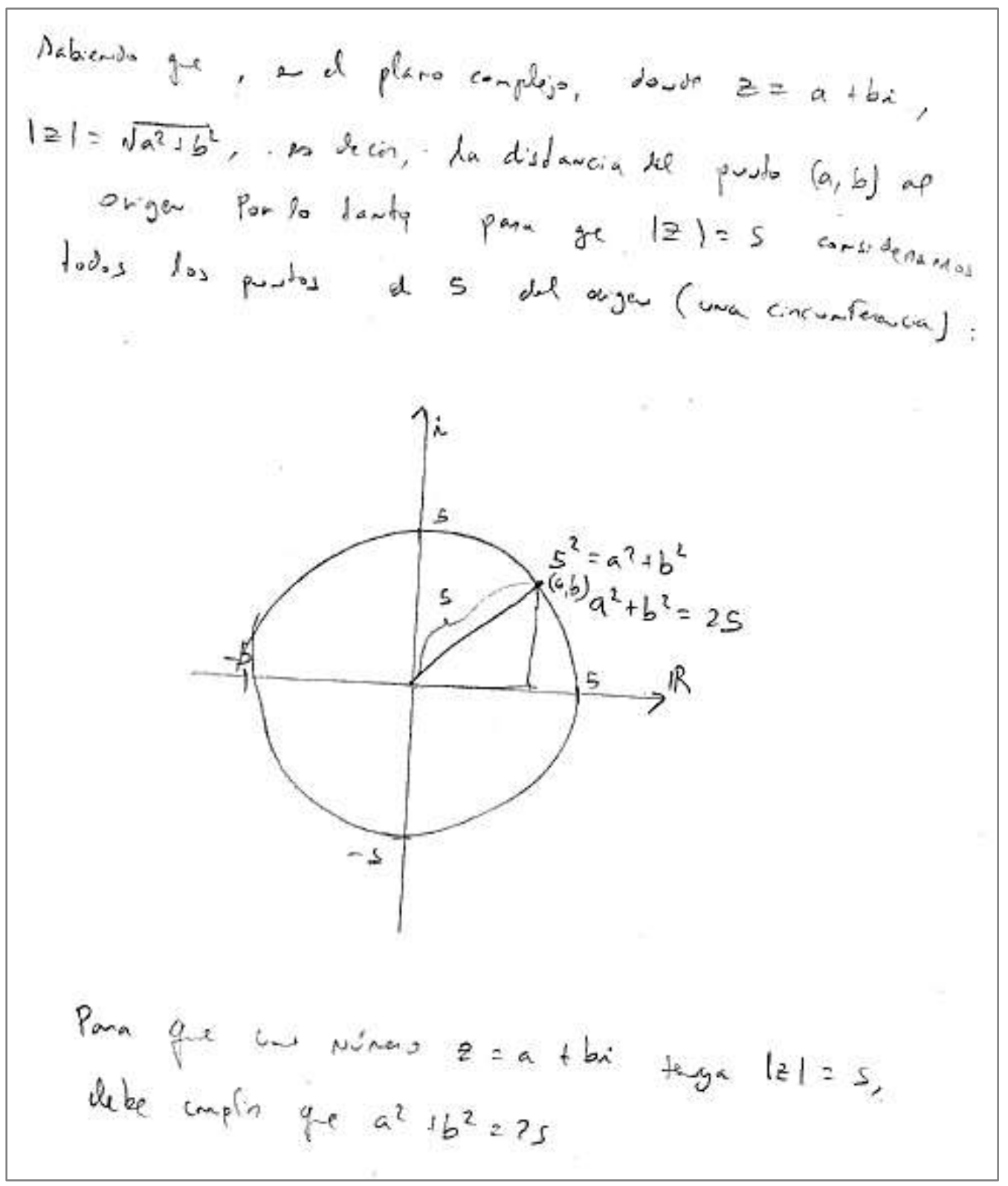

Fig. 6: Respuesta de E6 a A2. 
Tabla 6: Respuestas de E2 y E4 para A3.

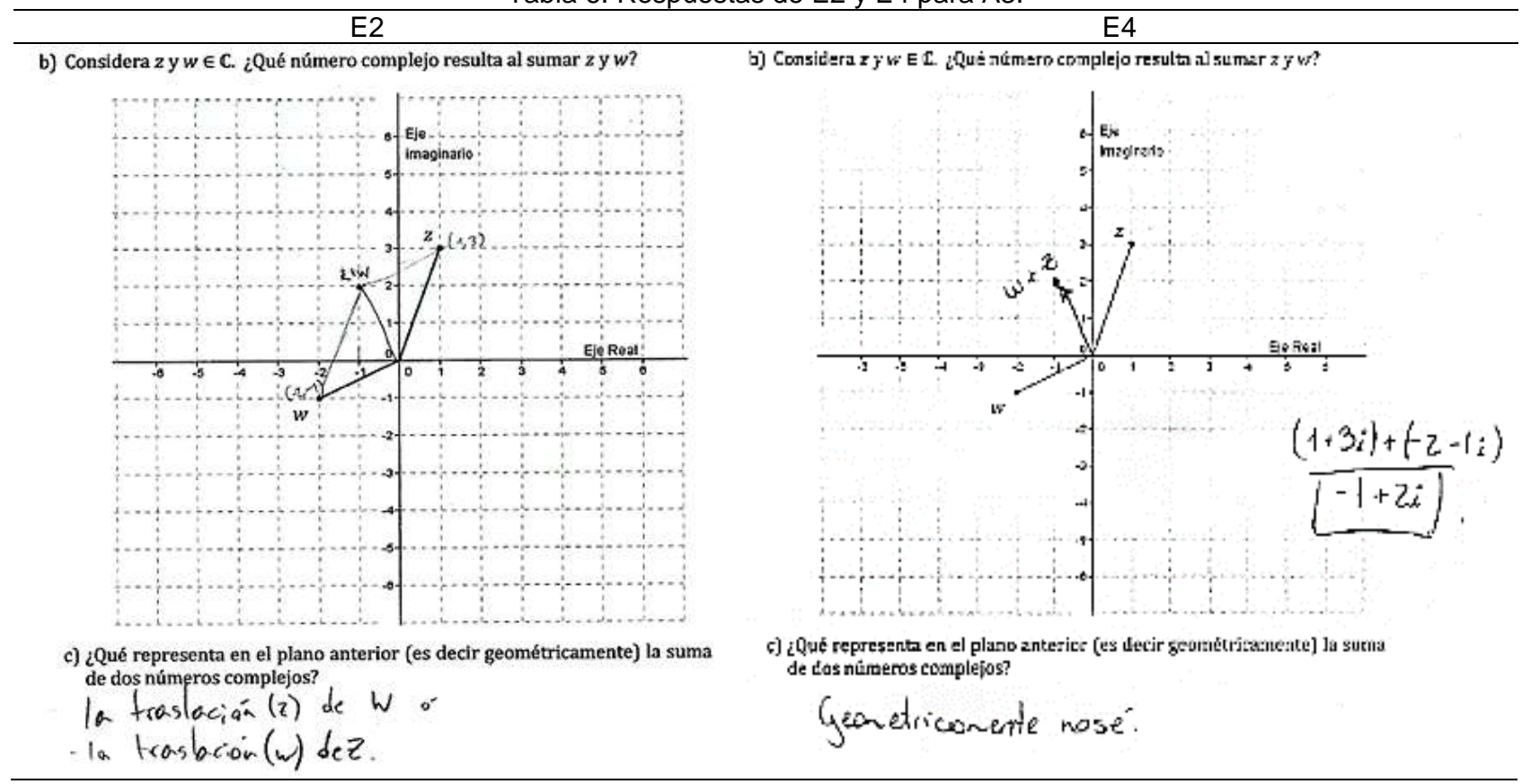

3. Carencia de tránsito hacia el modo AE-SNC: Al mismo tiempo que los estudiantes están situados en el modo AA-SNC, se evidencia que no hay articulación hacia el modo AE-SNC. Los informantes desconocen elementos de la estructura algebraica del sistema numérico, tales como propiedades y axiomas, salvo $i^{2}=-$ 1, la que utilizan implícita o explícitamente cuando operan números complejos. De acuerdo con esto, ellos están entendiendo a los números complejos como un conjunto de elementos que cumple ciertas "fórmulas". En la Fig. 7 el estudiante E7 entiende a los números complejos como un conjunto más grande que los números reales, pero aparte de ellos lo cual es confuso. En la Fig. 8, se puede ver también como el informante E10 en la A9 no acepta a $\sqrt{ }-1$ como solución de la ecuación $x^{2}=-1$ por que utiliza la propiedad $\sqrt{ } a \cdot b=\sqrt{ } a \cdot \sqrt{ } b$, que no es cierta en el cuerpo de los números complejos.

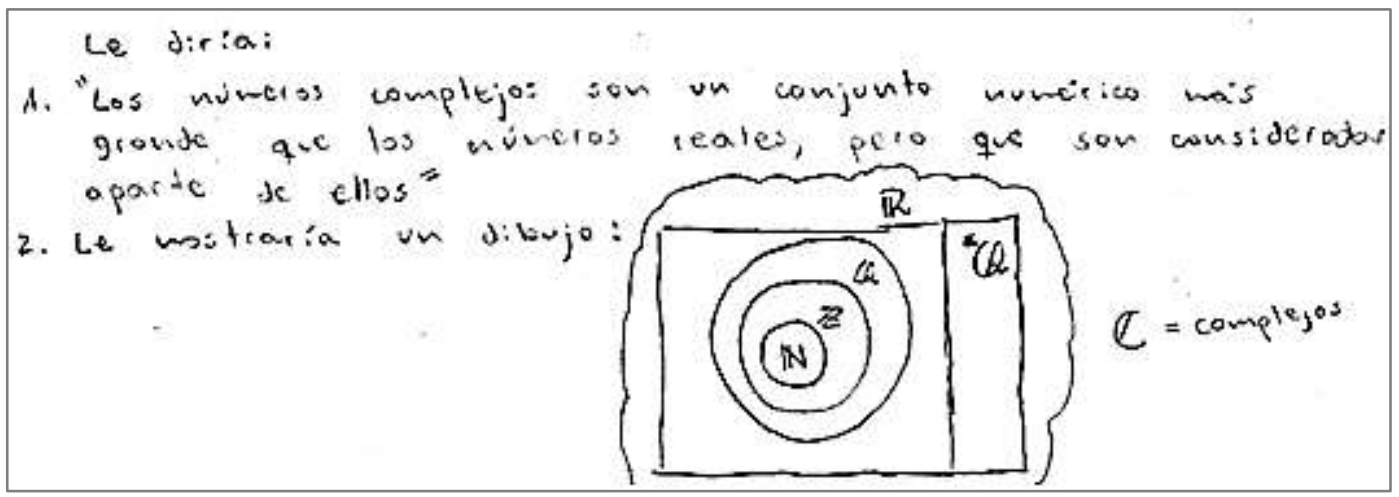

Fig. 7: Respuesta de E7 a P2.

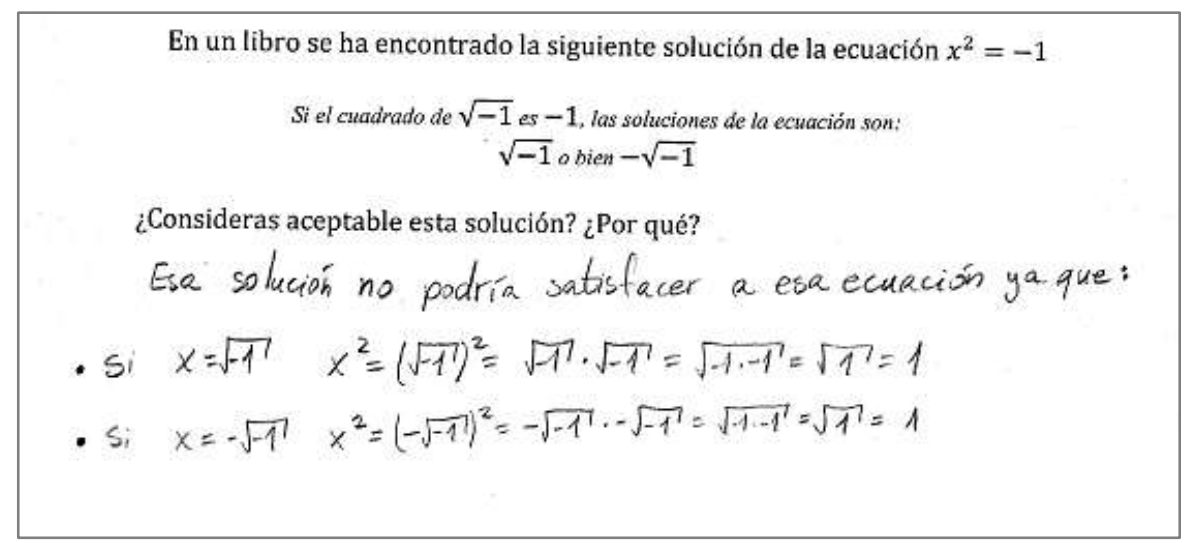

Fig. 8: Respuesta de E10 a A9. 


\section{Hallazgos para el Caso III: futuros profesores de matemática}

4. Privilegio por AA-SNC y presencia de dificultades: Los estudiantes de pedagogía en matemática, priorizan un pensamiento AA-SNC en varias de las actividades y presentan algunas dificultades. Por ejemplo, algunos informantes en la A4 no recordaron cómo realizar la división de dos números complejos y otros cometieron errores de cálculo. Si bien los informantes de este caso privilegian en mayor medida el modo SG-SNC que los Casos I y II, hay una tendencia a preferir el pensamiento AA-SNC. En la A2, por ejemplo, solo uno de los estudiantes logra resolver con éxito la ecuación, al ser el único que articula un pensamiento AA-SNC con uno SG-SNC; por su parte el resto de los informantes solo dio como respuesta casos particulares, lo cual es insatisfactorio para su nivel de conocimientos (Fig. 9).

$\begin{aligned} &|a+b i|=5 \\ & \sqrt{a^{2}+b^{2}}=5 \\ & a^{2}+b^{2}=25 \\ & a^{2}=25-b^{2} \\ & a=\sqrt{25-b^{2}} \\ & a \in \mathbb{R}[-5 \cdot 5]\end{aligned}$

Fig. 9: Respuesta de E13 a A2 desde el modo de pensar AA-SNC.

5. Hay tránsitos desde y hacia SG-SNC: Los informantes de este caso de estudio se sitúan en SG-SNC, pero no alcanzan este modo de pensar totalmente. En las preguntas iniciales del cuestionario señalan el plano complejo dentro de sus definiciones, al contrario de los casos anteriores. Además, los estudiantes utilizan estrategias geométricas para realizar la operación suma (Fig. 10), pero solo manejan técnicas mecánicas desde AA-SNC para la operación multiplicación. Se evidenció, por ejemplo, que un estudiante utiliza la notación $r e^{i \theta}$ para hacer multiplicaciones en la A5, aplicando propiedades de potencias: $r e^{i \theta} \cdot t e^{i \mu}=r t e^{i(\theta+\mu)}$ (Fig. 11). En este sentido, el modo de pensar SG-SNC no es alcanzado cabalmente.

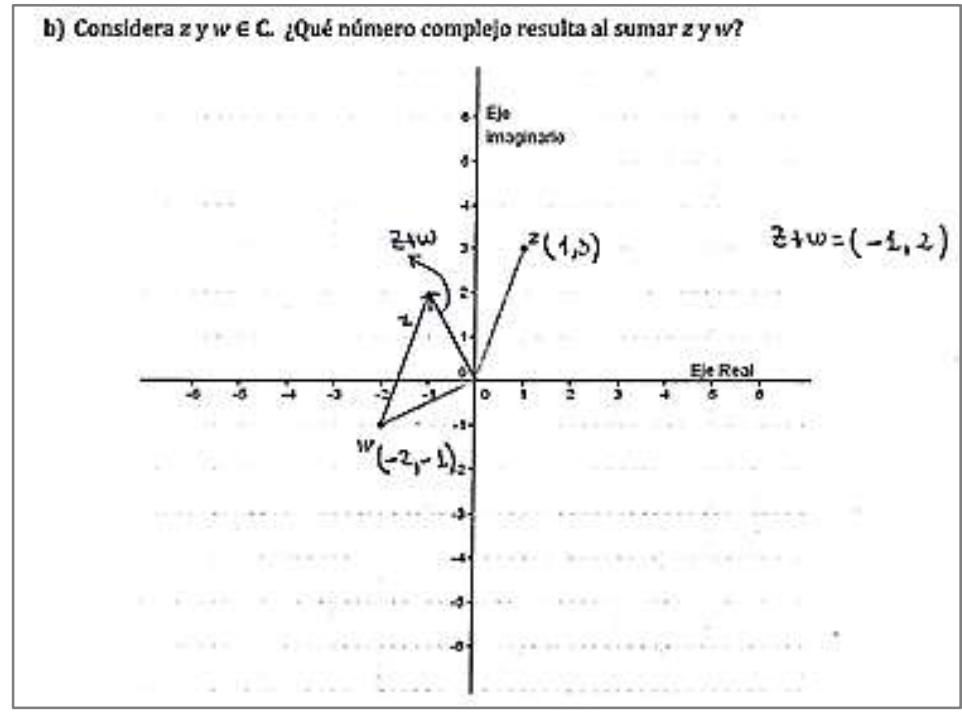

Fig. 10: Respuesta de E15 a A3 desde SG-SNC.

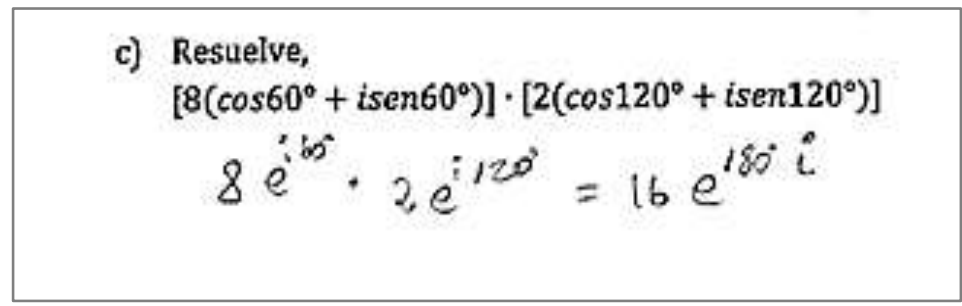

Fig. 11: Respuesta de E12 a A5 c). 
Al tener mayores conocimientos que los llevan a situarse en el modo SG-SNC, se logran ver tránsitos y articulaciones entre los modos SG-SNC y AA-SNC. Por ejemplo, en la A10 los informantes articulan los modos SG-SNC y AA-SNC en la necesidad de visualizar el problema en el plano complejo utilizando argumentos en términos de transformaciones (reflexión). En la Fig. 12, por ejemplo, se presenta el desarrollo del informante E14, quien comienza resolviendo desde un modo de pensar SG-SNC y luego transita hacia el modo AA-SNC mediante la ecuación de la circunferencia, pero se equivoca al considerar solo la parte imaginaria de $z$, ya que se confunde cuando anota $z \in \mathbf{C}$, que lo lleva a descartar la parte real del número.

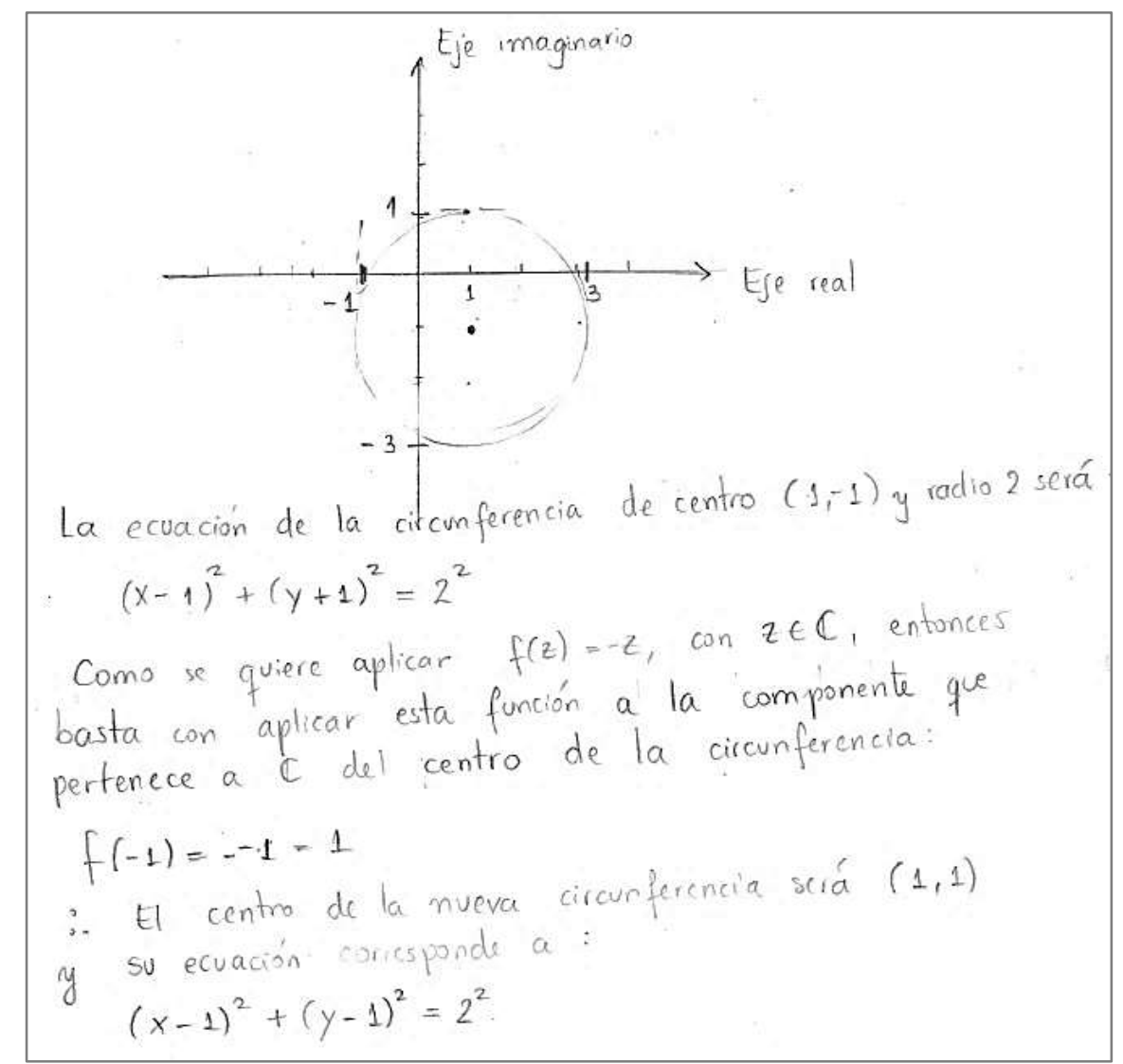

Fig. 12: Respuesta de E14 a A10 haciendo el tránsito SG-SNC $\rightarrow$ AA-SNC.

6. El pensamiento AE-SNC es alcanzado, pero faltan articulaciones: los futuros docentes se sitúan en un modo de pensar AE-SNC, aunque no se observa articulación hacia el modo SG-SNC. Los informantes conocen y utilizan la propiedad fundamental $i^{2}=-1$ y lo conectan con el modo AA-SNC para resolver las actividades. El informante E13 (Fig. 13), por ejemplo, responde desde un modo de pensar AE-SNC a la actividad seis a) i., utilizando el teorema de De Moivre para calcular la potencia y articular así el teorema (AE$\mathrm{SNC}$ ) con la operatoria a través de la notación polar del número complejo (AA-SNC); en el ítem a) ii. solo calcula $i^{100}$ y no encuentra el valor de la suma.

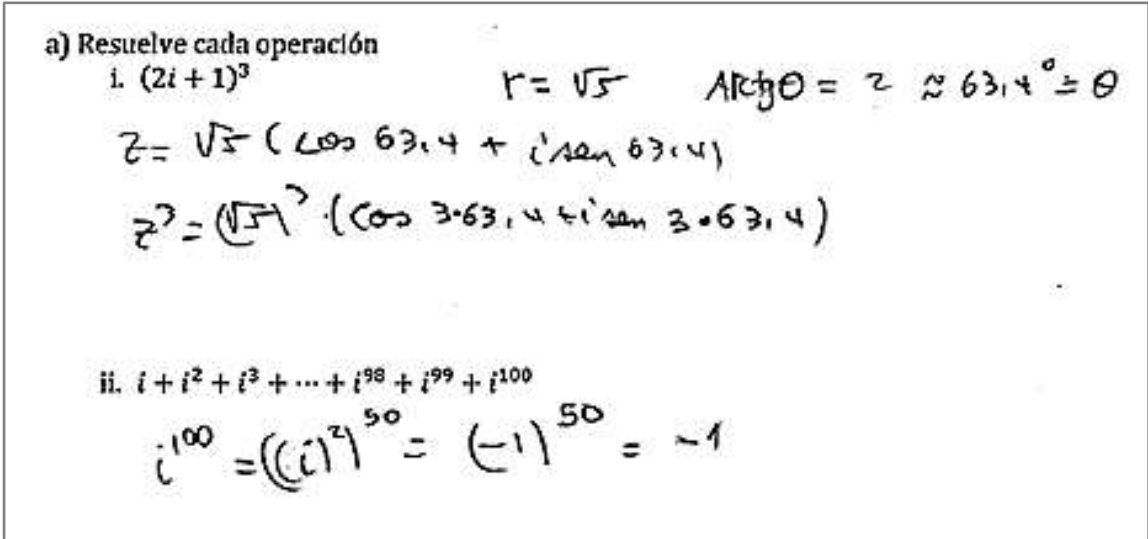

Fig. 13: Respuesta de E13 a A6 haciendo el tránsito AE-SNC $\rightarrow$ AA-SNC. 
También, los estudiantes de pedagogía reconocen la estructura de orden del sistema numérico, señalando que los números complejos no se pueden ordenar como los números reales. Algunos informantes son muy específicos en su argumentación, pero otros no. En la Tabla 7, se muestra la respuesta de E12 y E14. Por un lado, E12, señala que hay un problema con $i$ y que no habría forma de ordenarlos, lo que no es completamente cierto ya que se podría definir un orden lexicográfico, por ejemplo; por otro lado, E14 muestra la propiedad de orden de los números reales que no cumplen los números complejos y da un contraejemplo suponiendo que $i>0$ e $i<0$.

Tabla 7: Respuestas de E12 y E14 para P5 sobre la estructura de orden del SNC. E14
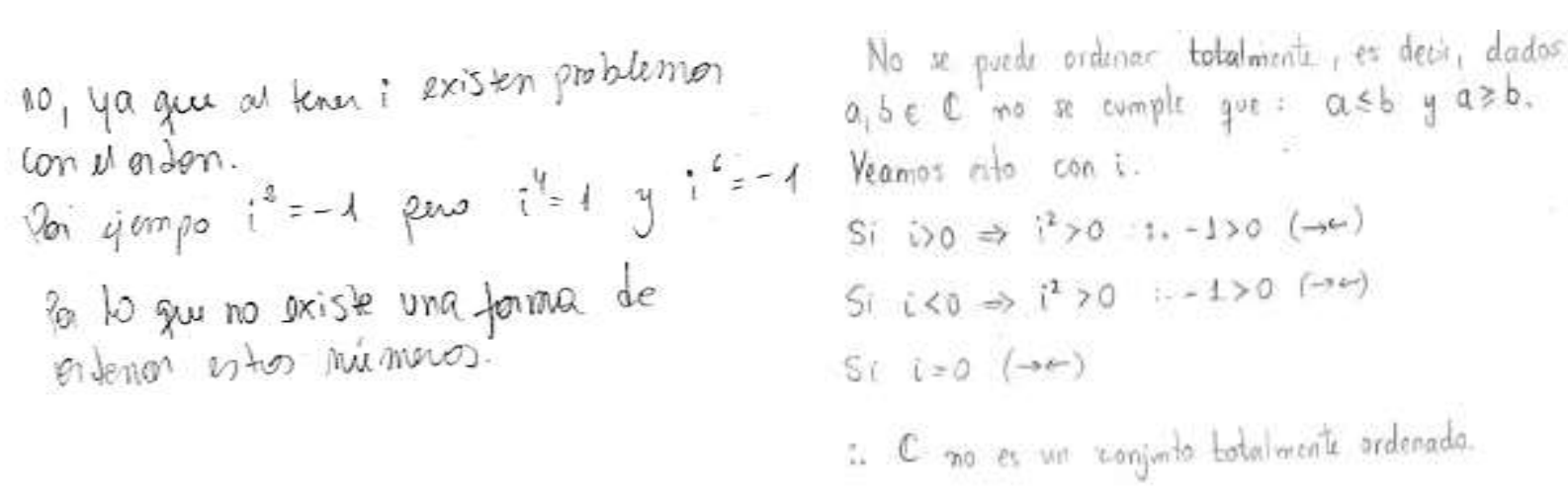

En la A7, donde se esperaba ver la articulación $\mathrm{AE}-\mathrm{SNC} \rightarrow \mathrm{AA}-\mathrm{SNC} \rightarrow \mathrm{SG}-\mathrm{SNC}$, a través del cálculo de las raíces cúbicas de -1 , se evidenció precisamente que $A E-S N C$ no está completamente alcanzado por los estudiantes ya que dos de ellos no respondieron a la actividad y de los otros dos informantes, solo uno de ellos, E15, realizó el tránsito correctamente a SG-SNC, como se puede ver en la Fig. 14, donde el estudiante contesta desde un modo de pensar AE-SNC que articula con AA-SNC en el cálculo de operaciones y luego en el ítem b) transita a SG-SNC señalando que se formará un triángulo equilátero en el plano complejo, incluso es capaz de generalizar este resultado para las raíces quintas de -1 donde indica que se formará entonces un pentágono regular.

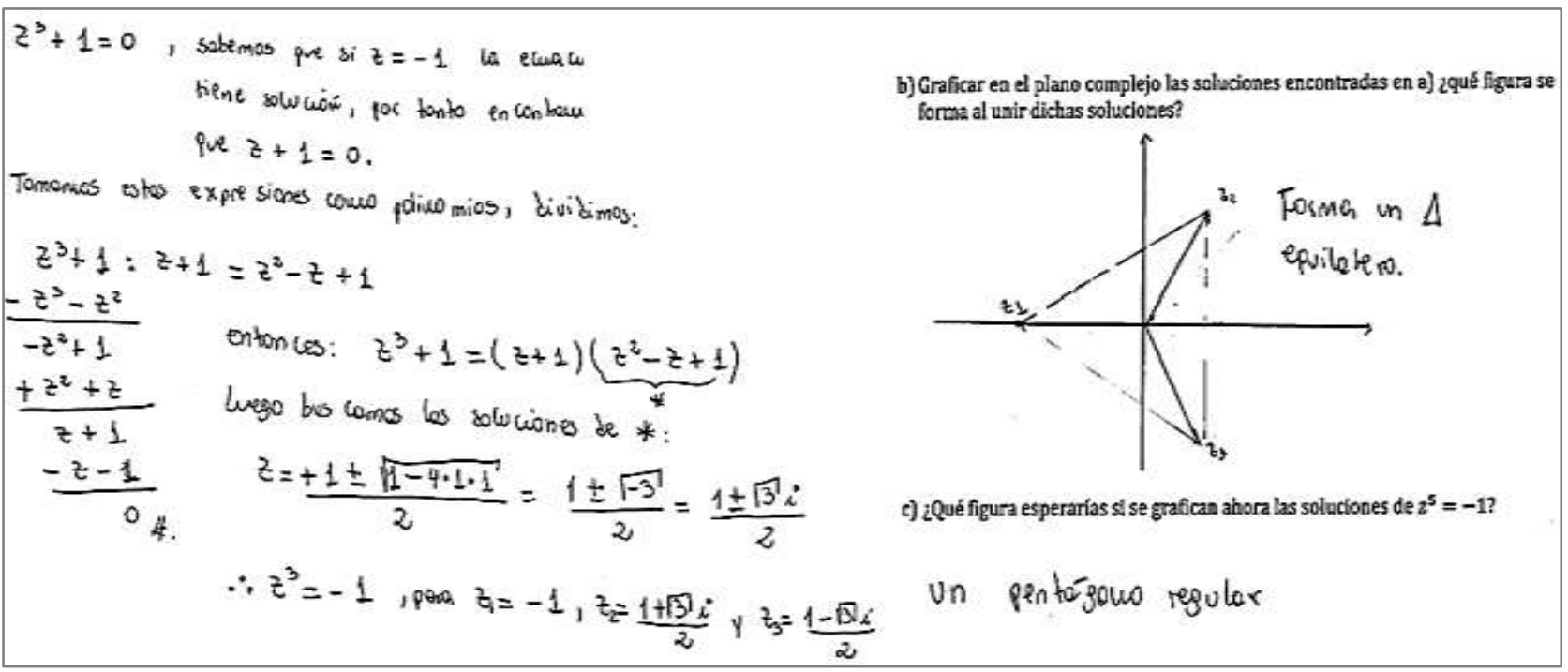

Fig. 14: Respuesta de E15 a A7 haciendo el tránsito AE-SNC $\rightarrow$ AA-SNC $\rightarrow$ SG-SNC.

\section{Hallazgos para el Caso IV: estudiantes de licenciatura en matemática}

Los informantes de este caso dan cuenta de situarse en los modos SG-SNC, AA-SNC y AE-SNC, aunque no alcanzan a cabalidad el modo SG-SNC y AE-SNC. Si bien evidencian mayor tránsito entre los modos y conexiones como AE-SNC $\rightarrow$ AA-SNC, presentan un privilegio por el modo AA-SNC en algunas actividades y carencia de conocimientos respecto al modo $\mathrm{AE}$, que no corresponden a su nivel de conocimientos.

7. Privilegio por AA: En algunas actividades, los estudiantes de último año de licenciatura se sitúan solo en un modo de pensar AA-SNC para responder. Por ejemplo, E17 desde AA-SNC solo lista casos particulares en la resolución de la A2; mientras que E16, responde a la multiplicación de dos números complejos (A4) solo 
desde el modo AA-SNC. La A10, por su parte, tuvo menor éxito que el caso anterior, ya que los informantes privilegiaron el modo AA-SNC y no SG-SNC. El informante E16, por ejemplo, utiliza la notación de conjuntos $|z-c|=r$, dejando expresada su respuesta como $f(A)$ (Fig. 15).

$$
\begin{gathered}
|z-(1-i)| \leq 2 \\
A=\{z<\phi|| z+1-i \mid=2\} \\
f(A)=\{f(z) \mid z \in A\}=\{-z /|z+1-i|=2\}
\end{gathered}
$$

Fig. 15: Respuesta de E16 a A10.

8. El modo SG-SNC está más presente en las respuestas: En cuanto al modo SG-SNC, los informantes patentizan en sus respuestas la utilización del plano complejo (Fig. 16), pero no ven la dinámica de las operaciones en él. Tal como sucede con el resto de los casos, la multiplicación de números complejos no es trabajada geométricamente (Fig. 17).

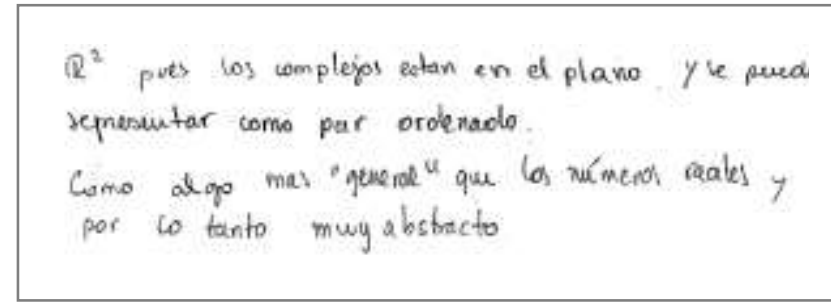

Fig. 16: Respuesta de E16 a P1.

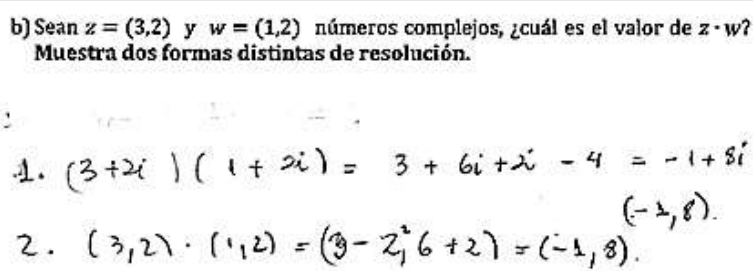
Muestra dos formas distintas de resolución.

1. $(3+2 i)(1+2 i)=3+6 i+2 i-4=-1+8 i$

$(3,2) \cdot(-1,2)=\left(3-2^{2}\right)$$$
(-
$$

Fig. 17: Respuesta de E16 a A4.

Los estudiantes dan cuenta, además, del tránsito SG-SNC $\rightarrow$ AA-SNC en la actividad tres, cuando suman los números complejos geométricamente e indican que correspondería a la diagonal del paralelógramo formado (Fig. 18).

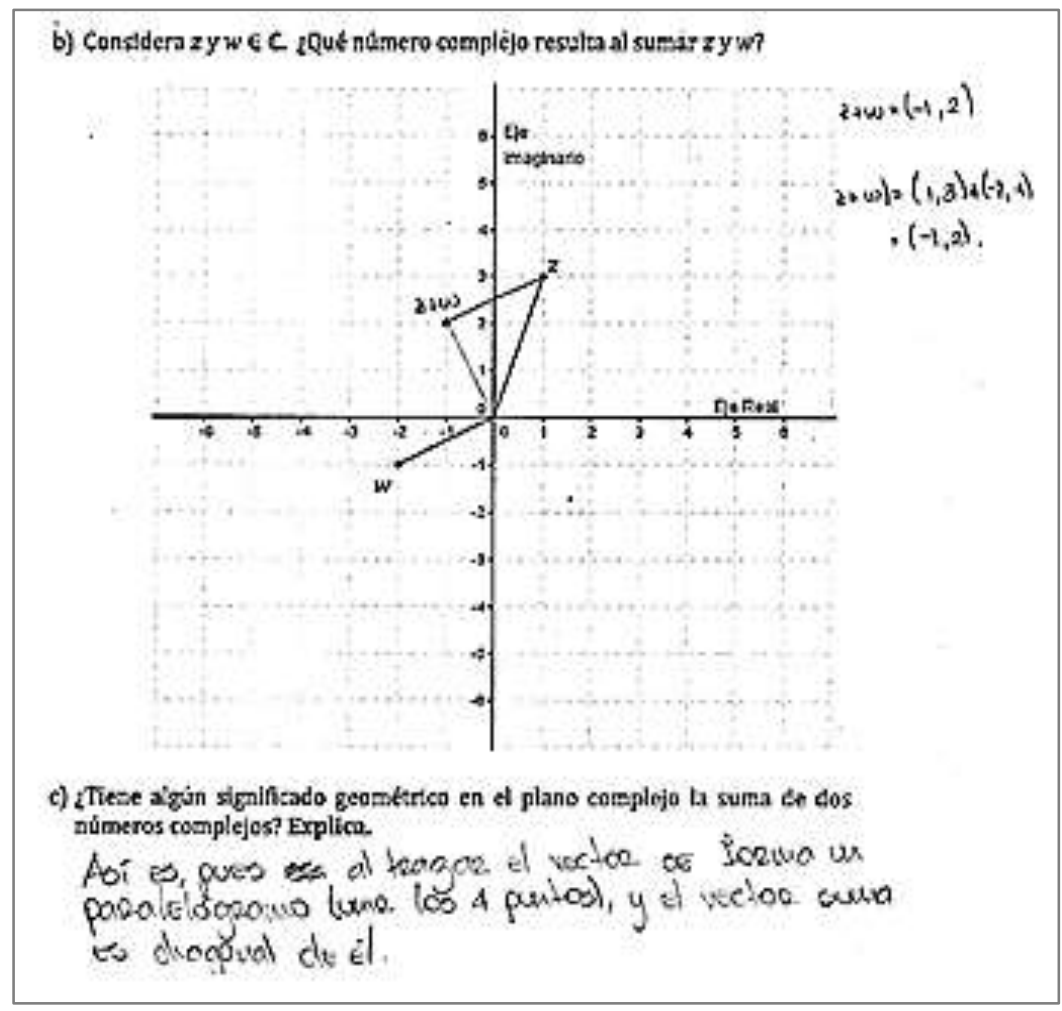

Fig. 18: Respuesta de E17 a A3.

Por otro lado, en la actividad ocho también se sitúan en un modo de pensar SG-SNC para argumentar que $i=(0,1)$, al contrario de los casos anteriores que lo hacían desde AA-SNC. La Fig. 19 muestra la respuesta de E16 a esta actividad. 


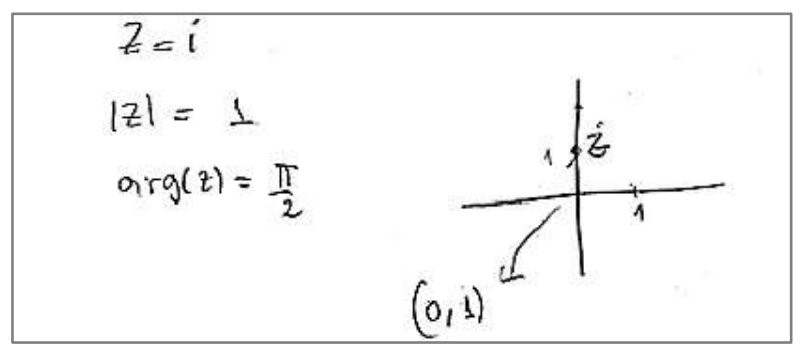

Fig. 19: Respuesta de E16 a A8.

9. Hay tránsitos desde y hacia AE-SNC, pero faltan elementos: Los informantes mostraron falta de argumentos para explicar que el SNC no es un sistema numérico ordenado como los números reales. Cabe destacar que el SNC no es un cuerpo que conserve la estructura de orden de $\mathbf{R}$, debido a que no satisface el postulado siguiente: $a>0, b>0 \Rightarrow a b=0$ (basta tomar $i>0$ ó $-i>0$ y llegar a una contradicción). E16, por ejemplo, se refirió a que no hay menor elemento, lo que es incompleto, pues por ejemplo el sistema de los números enteros no tiene menor elemento, sin embargo, presenta un orden que es el mismo que el del sistema de los números naturales. A pesar de ello, definen $i$ tal que $i^{2}=-1$ como propiedad fundamental del cuerpo y distinguen el desarrollo de todo un cálculo para el SNC desde la definición de la variable compleja, como se muestra en la Fig. 20.

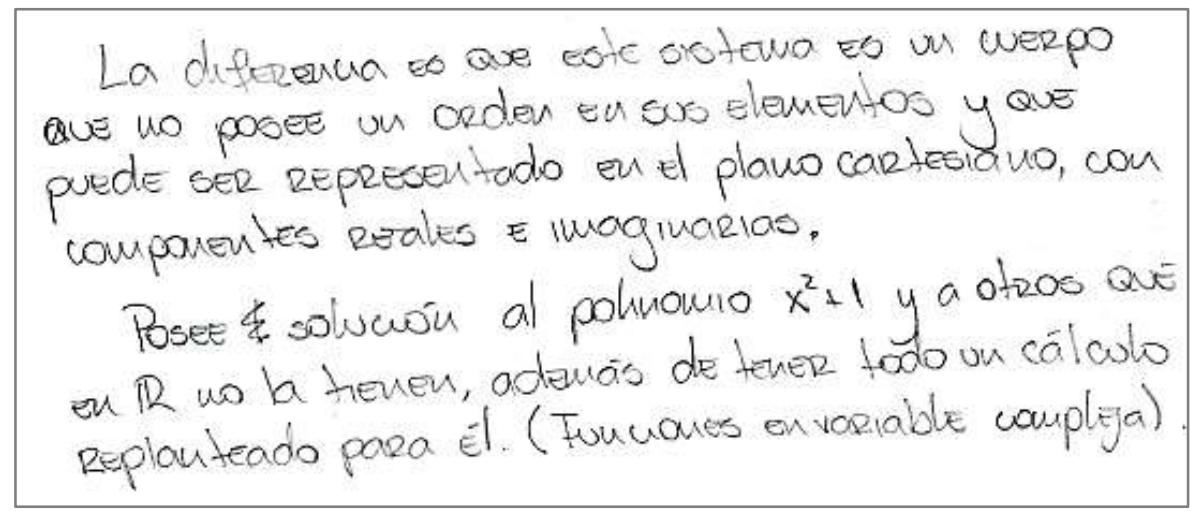

Fig. 20: Respuesta de E17 a P3.

Además, evidencian el tránsito $\mathrm{AE}-\mathrm{SNC} \rightarrow \mathrm{AA}-\mathrm{SNC} \rightarrow \mathrm{SG}-\mathrm{SNC}$ en la actividad siete, al calcular las raíces cúbicas de -1. En la Fig. 21, E17 responde desde un modo de pensar AE-SNC utilizando el Teorema Fundamental del Álgebra que le ayuda a reconocer tres raíces para esa ecuación; luego, mediante operaciones desde la notación $r e^{i \theta}$ calcula las raíces correctamente, transitando a AA-SNC. Para el ítem b), responde desde un modo de pensar SG-SNC, notando que las raíces forman los vértices de un triángulo equilátero; y que las raíces de la ecuación $z^{5}=-1$, por tanto, formarán un polígono regular de cinco lados.

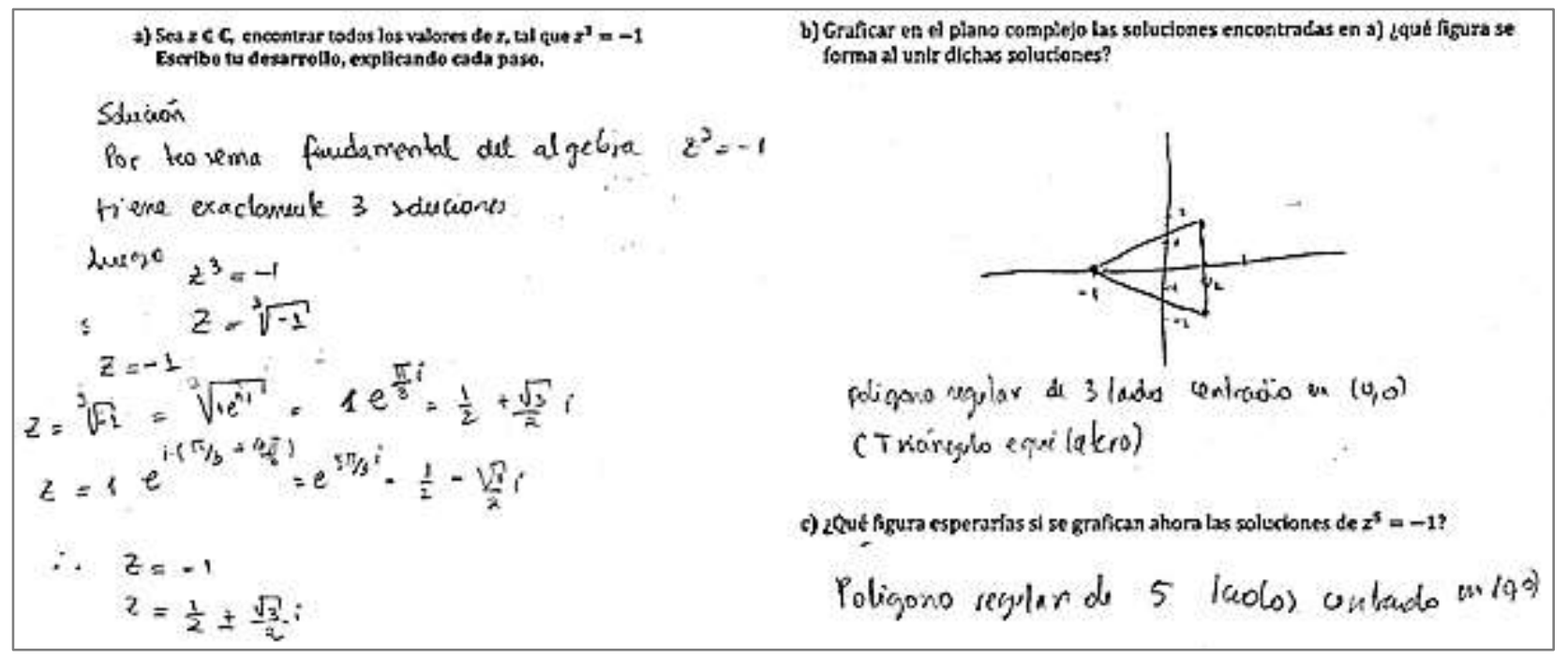

Fig. 21: Respuesta de E17 en A7. 


\section{Hallazgos para el Caso V: estudiantes de magister y doctorado en matemática}

Los informantes de este grupo muestran en sus argumentos alcanzar los modos SG-SNC, AA-SNC y AESNC y de transitar articuladamente entre ellos en el desarrollo de las actividades, lo que permite identificar elementos articuladores entre los modos para movilizar el pensamiento en cuanto a la comprensión profunda del SNC.

10. Privilegio por el modo SG-SNC: Los informantes de este caso, privilegian SG-SNC en varias de las actividades y muestran elementos geométricos tales como multiplicar por $i$ es rotar en $\pi / 2$; que el módulo se define como la distancia del número complejo al origen del plano complejo; y que al multiplicar dos números complejos se están multiplicando sus módulos y sumando sus argumentos, en coordenadas polares. Éstas últimas juegan un rol relevante en lo presentado por los informantes ya que las utilizan en casi todas las actividades. En la Fig. 22, E20 define a al sistema numérico desde SG-SNC, señalando que cada número complejo será un punto en el plano complejo.

$$
\begin{aligned}
& \text { En primir lugar, veo un plano } \mathbb{R}^{2} \text { y cado punto loveo } \\
& \text { como un número complejo y empiezo a visializar toda } \\
& \text { la dinómica que hacen los propiedadcs de ellos. }
\end{aligned}
$$

Fig. 22: Respuesta de E20 en P1.

El informante E19, también resuelve desde el modo SG-SNC en la A10, pensando en que multiplicar por menos uno significa rotar en 180 grados. Así, como se ve en la Fig. 23, resuelve la actividad señalando que basta con rotar el centro de la circunferencia dada.

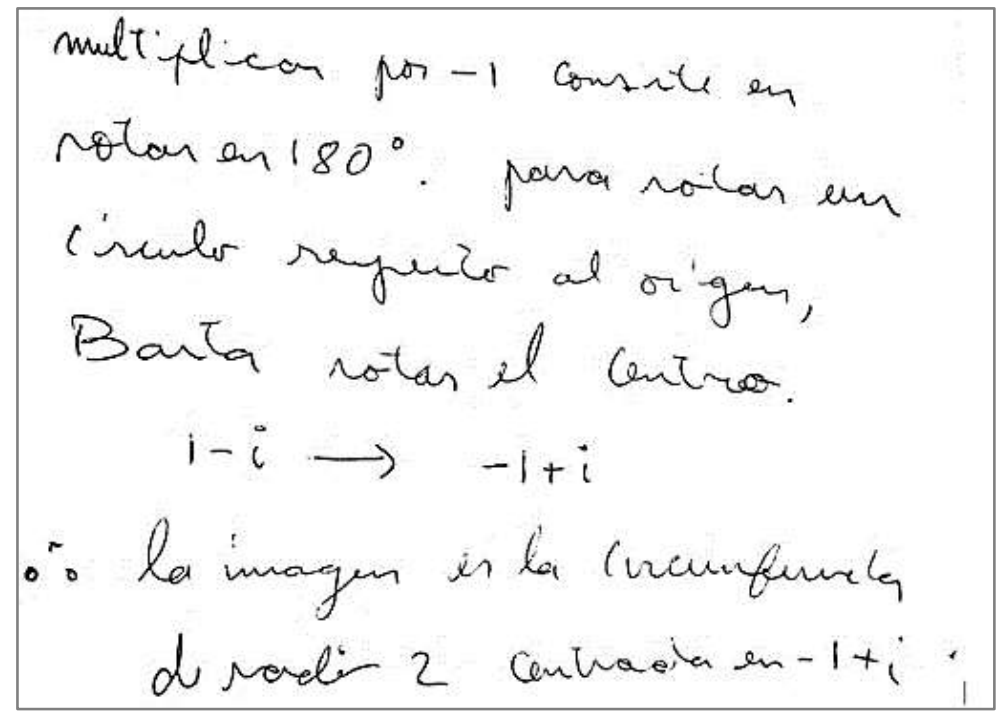

Fig. 23: Respuesta de E19 en A10.

Fue posible evidenciar, al mismo tiempo, el tránsito entre SG-SNC y AA-SNC. Por ejemplo, en la suma de números complejos algunos hicieron el tránsito SG-SNC $\rightarrow$ AA-SNC y otro AA-SNC $\rightarrow$ SG-SNC. En este sentido, los informantes entendían la suma tanto geométrica en el plano, como algebraica con las notaciones binomial o de par ordenado. Por otro lado, la multiplicación de números complejos que solo ha sido presentada desde AA-SNC por los informantes de los casos anteriores, fue señalada desde la notación $r e^{i \theta}$, articulando una idea geométrica, que es dibujada en un costado de la hoja, con cálculos numéricos (Fig. 24).

11. AA-SNC y tránsitos hacia los otros modos de pensar: En relación con el modo de pensar AA-SNC, los informantes calculan utilizando relaciones conocidas, pero justificando sus procedimientos. El informante E20 (Fig. 25), por ejemplo, no solo calcula las potencias de $i$ que se indican, sino que realiza una generalización utilizando la congruencia de números y transitando al mismo tiempo al plano complejo 


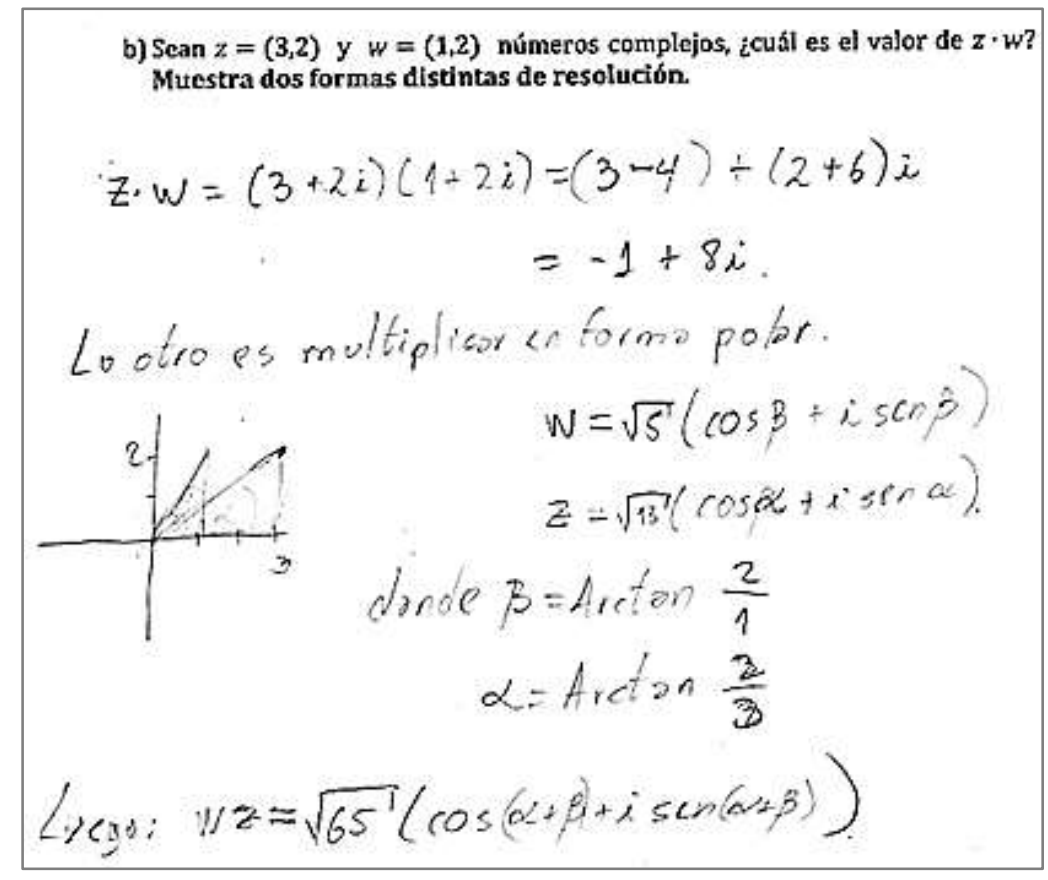

Fig. 24: Respuesta de E20 en A4.

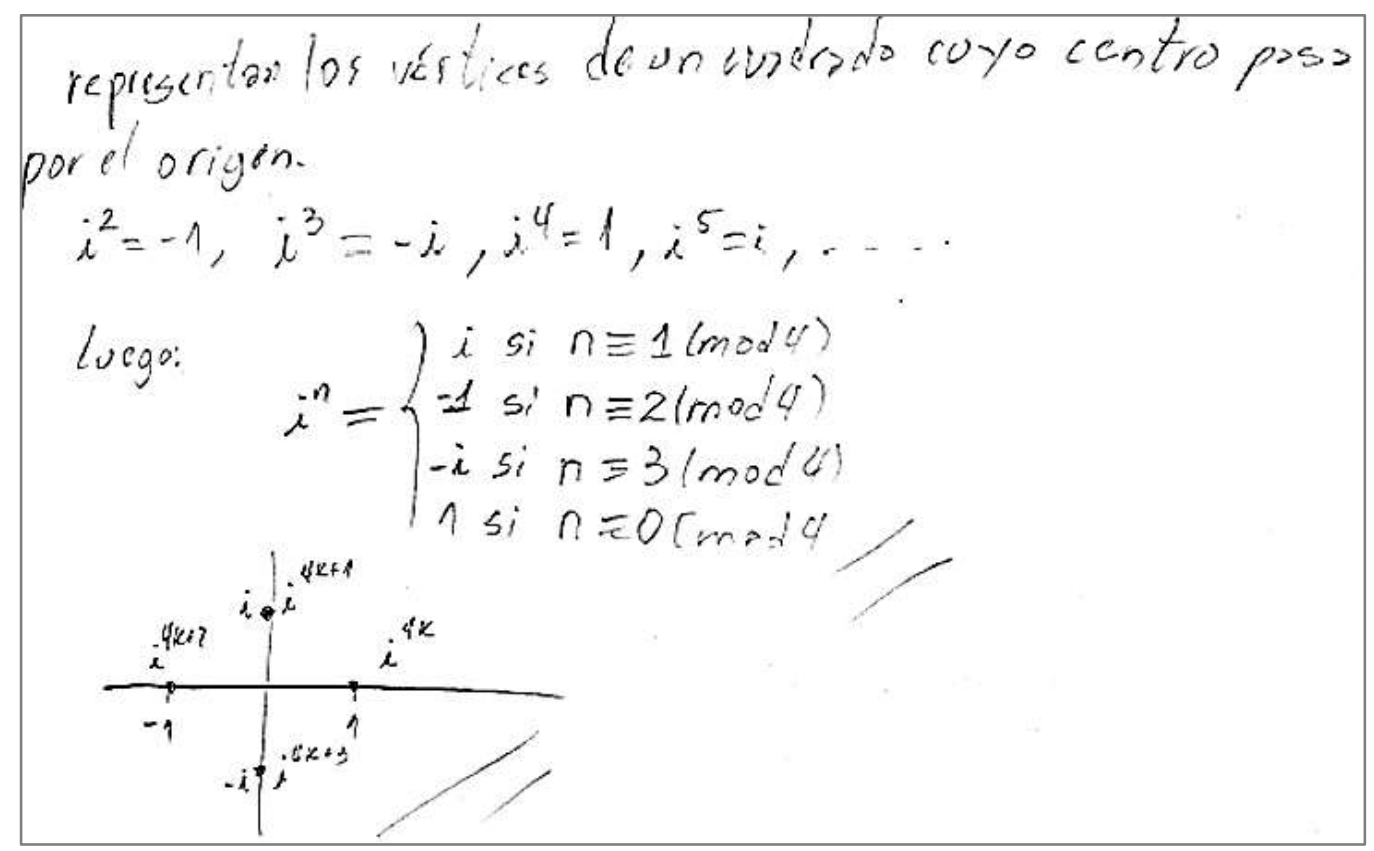

Fig. 25: Respuesta de E20 en A6.

La notación $r e^{i \theta}$ es utilizada con frecuencia en las actividades (Fig. 26) y no solo muestra ser una manera más sencilla de realizar cálculos, sino de transitar entre los modos de pensar, ya que $(r, \theta)$ tiene una interpretación directa en el plano complejo.

12. El pensamiento AE-SNC es alcanzado: Por su parte para el modo AE-SNC, los informantes se sitúan sin dificultades y lo alcanzan. Se refieren tanto a propiedades como $i^{2}=-1$ que define al cuerpo, como a la clausura algebraica, al Teorema Fundamental del Álgebra y al desarrollo de la variable compleja. Además, dan cuenta de entender en profundidad la estructura de orden del sistema numérico. El informante E20, por ejemplo, indica que sí es posible ordenar a los números complejos, pero no con las propiedades de orden de los números reales, sino que se podría definir uno, por ejemplo, lexicográfico, usando desigualdades que cumplan la condición $a+b i \leq c+d i \leftrightarrow[a \leq c \vee(a=c \wedge b \leq d)]$. También es posible evidenciar tránsitos desde y hacia AE-SNC. En la A7, por ejemplo, se articulan los modos AA-SNC y AE-SNC, y luego con SG-SNC, 
donde se aplica el Teorema de De Moivre para calcular las raíces cúbicas de menos uno, que luego se dibujan en el plano complejo (Fig. 27).

$$
\begin{aligned}
& \text { Forma I: Si la forma polar de } z \text { en } r e^{1 \theta} \text {, } \\
& \begin{aligned}
5=\left|r e^{i \theta}\right|=|r|\left|e^{i \theta}\right|=r|\cos \theta+i \sin \theta| & =r \sqrt{\cos ^{2} \theta+\sin ^{2} \theta} \\
& =r .
\end{aligned} \\
& \text { como la condición de } r \text { no depende de } \theta \text {, } \\
& \text { teremos quel el coujunto solución es. } \\
& S=35 e^{i \theta}, \theta \in[0,2 \pi[\} \text {. } \\
& \text { Forma } \pi \text { : Si } z=\operatorname{Re}(z)+\operatorname{Im}(z) i \text {, eutonus quiero } \\
& \begin{array}{l}
\text { quenes cumpleu con que } \\
\qquad|z|=\sqrt{\operatorname{Re}(z)^{2}+\operatorname{Im}(z)^{2}}=5 .
\end{array} \\
& \text { - equiralentemente, } \\
& \operatorname{Re}(z)^{2}+\operatorname{Im}(z)^{2}=5^{2} \\
& \text { I notanos que los puntor) (PC }(z) \text {, Tru }(z) \text { ) en el } \\
& \text { plano complejo satisfacen la ecuación de un } \\
& \text { circun ferencia de radio } 5 \text { : }
\end{aligned}
$$

Fig. 26: Respuesta de E18 en A2.

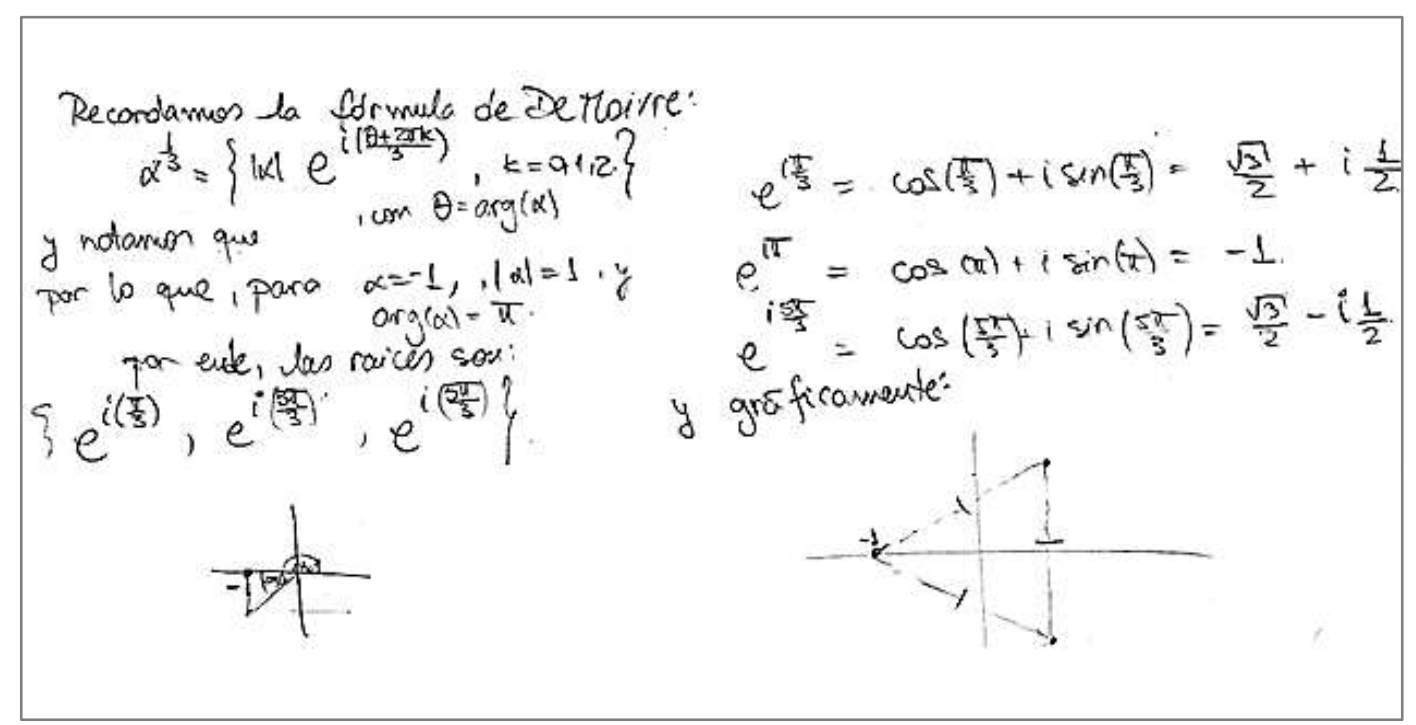

Fig. 27: Respuesta de E18 en A7.

\section{SUGERENCIAS DIDÁCTICAS}

Al respecto de cómo es posible alcanzar la comprensión profunda del SNC, las evidencias entregadas por los informantes del Caso V, sumados a los análisis epistemológico y matemático del objeto de estudio permiten argumentar que los elementos de la matemática que promueven el tránsito entre los modos de comprender el SNC obedecen a ciertos conceptos y estructuras descritos en la Tabla 1 y que se presentan en el modelo de la Fig. 28, como elementos articuladores entre los modos de pensar el SNC, describiendo la interacción completa de los elementos que constituyen el modelo de su comprensión. Con base en este modelo es posible realizar algunas sugerencias para el diseño de actividades y situaciones de aprendizaje. 
Tránsito SG-SNC $\leftrightarrow A A-S N C$ : Para que los aprendices puedan hacer el tránsito SG-SNC $\leftrightarrow$ AA-SNC es recomendable la presentación del sistema numérico como puntos $(a, b)$ del plano complejo. Esto les permitirá trabajar con ambas representaciones haciendo una correspondencia de puntos. Para la dirección AA-SNC $\rightarrow$ SG-SNC, por su parte, se sugiere el empleo de coordenadas polares. El uso de la forma $r e^{i \theta}$, por ejemplo, permite acceder a las variables ángulo y distancia (módulo), que conectan con el plano complejo en las operaciones suma y producto mediante movimientos en el plano, ya que desde el desarrollo geométrico del SNC se establece un sistema distinto del cartesiano para establecer coordenadas de cada punto del plano: a cada número complejo $z$ corresponde un par de coordenadas polares $(r, \theta)$, del vector asociado, tal que $z=$ $a+b i=(a, b)=r(\cos (\theta)+i \operatorname{sen}(\theta))=r e^{i \theta}=(r, \theta)$. Su empleo permite que algunas propiedades algebraicas sean más transparentes y que los cálculos sean más sencillos y eventualmente más poderosos (como se ha visto en este trabajo). La transformación rotación tiene especial relevancia en este sentido, pues a nivel de sistemas numéricos tal movimiento solo es posible de hacer en el SNC. Un ejemplo es notar que la multiplicación por $i$ produce una rotación del vector asociado en $\pi / 2$ radianes. De este modo, se pueden proponer actividades como A2 y A10, pero orientadas al trabajo en SG-SNC.

Tránsito $A A-S N C \leftrightarrow A E-S N C$ : Transitar desde y hacia $A E-S N C$ no es sencillo y requiere de nociones matemáticas más avanzadas. Para el tránsito AE-SNC $\rightarrow$ AA-SNC la propiedad $i^{2}=-1$ y teoremas y propiedades, como el teorema de De Moivre y el teorema Fundamental del Álgebra, permiten a los aprendices moverse entre la realización de cálculos (como el de las raíces $n$-ésimas) y nociones de la estructura algebraica, de orden y topológica del SNC. Por su parte, la conexión AA-SNC $\rightarrow$ AE-SNC, según los hallazgos de esta investigación, requeriría pasar por un pensamiento SG-SNC, vale decir, que los aprendices entiendan a los números complejos como puntos del plano complejo (el plano cartesiano vestido ahora como el plano complejo), lo que los llevará hacia el isomorfismo con $\mathrm{R} \times \mathrm{R}$, visualizando la estructura de cuerpo. En este sentido, el tránsito que se debe propiciar para alcanzar AE-SNC desde AA-SNC, es AA-SNC $\rightarrow$ SG-SNC $\rightarrow$ AE-SNC. De ahí la importancia que los aprendices alcancen y transiten por modos SG-SNC y AA-SNC.

Tránsito $A E-S N C \leftrightarrow S G-S N C$ : Los articuladores que potenciaron este tránsito fueron algunas nociones ya mencionadas, como es el de entender a los números complejos desde su notación de pares ordenados; ésta posibilita establecer el isomorfismo, $\mathrm{C} \rightarrow \mathrm{R} \times \mathrm{R}$, conectando el $\mathrm{SNC}$ con $\mathrm{R}^{2}$, estructura que los aprendices conocen desde el nivel escolar. Las actividades que involucran raíces $n$-ésimas de un número complejo se sugieren para movilizar el pensamiento analítico-estructural al pensamiento sintético-geométrico del SNC debido a su relación con la representación de polígonos regulares en el plano complejo.

Los elementos involucrados en la comprensión profunda del SNC requieren de un conocimiento más profundo por parte de los estudiantes de educación escolar. Se espera que contenidos como las coordenadas polares, que también involucran a otras áreas de las ciencias, sean incorporadas y trabajadas en las unidades de números complejos. Además, se considera adecuado que el trabajo del sistema se inicie potenciando las conexiones SG-SNC $\leftrightarrow$ AA-SNC, para luego ir por las conexiones AA-SNC $\rightarrow$ SG-SNC $\rightarrow$ AE-SNC, pues AE-SNC es el modo más difícil de alcanzar para estos aprendices. De este modo, se sugiere potenciar actividades desde los modos SG-SNC y AE-SNC, situando operaciones en el plano complejo y definiendo a la unidad imaginaria desde $i^{2}=-1$ y no desde $i=\sqrt{ }-1$, dado que, a pesar de que no es una notación errada, los estudiantes tienden a aplicar propiedades que solo se cumplen en los números reales y no en los números complejos.

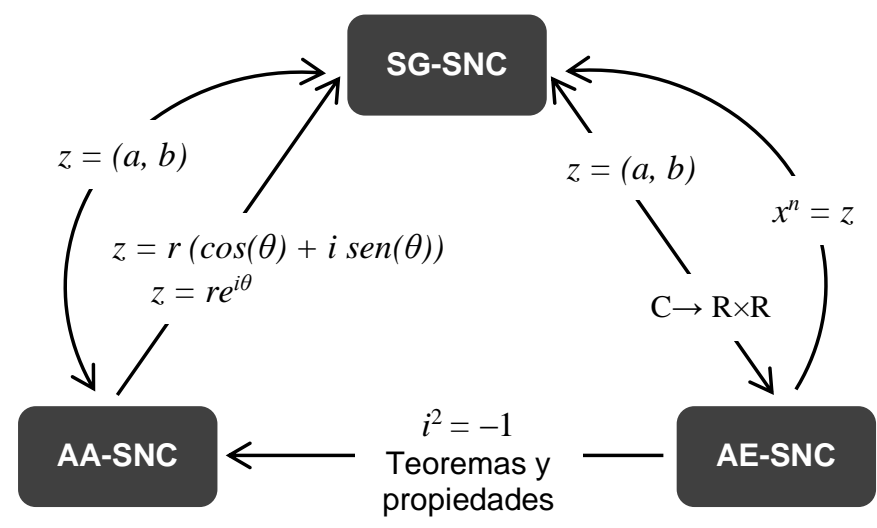

Fig. 28: Modos de pensar el SNC y articuladores validados en esta investigación. 


\section{CONCLUSIONES}

Desde los datos teóricos y empíricos que brindan los resultados de la investigación es posible concluir que para alcanzar una comprensión profunda del SNC es necesario conocer y transitar articuladamente por tres modos de pensar el sistema numérico: SG-SNC, AA-SNC y AE-SNC. Cada uno de estos modos brinda formas de ver y entender el sistema numérico, pero su coordinación y tránsito serán los que permitan a los aprendices acceder a un pensamiento más versátil que les conduzca a diferentes significados del objeto y por tanto a su comprensión cabal. Considerando esta premisa al analizar la comprensión del SNC en estudiantes escolares y universitarios chilenos, se concluye que éstos (1) en su mayoría, tienen una comprensión fragmentada del SNC debido principalmente a que privilegian y emplean el modo AA-SNC en la resolución de diferentes actividades, careciendo de tránsitos hacia y desde los modos SG-SNC y AE-SNC; y, al mismo tiempo y motivado por lo anterior, (2) entienden a los números complejos como un conjunto y no como un sistema numérico. En particular, es posible concluir para el caso de los estudiantes del nivel escolar, que éstos resuelven varias de las actividades desde los modos de pensar el sistema de los números reales y no desde los modos de pensar el SNC, dando cuenta de un sesgo de los números reales sobre los números complejos. Por ejemplo, ideas arraigadas desde los números reales, tal como insistir en que un número al cuadrado (o potencia par) es siempre positivo o en negar la raíz cuadrada de un número negativo, se manifiestan en el contexto de actividades que involucran al SNC. Por su parte, en el caso de los estudiantes universitarios futuros profesores de matemática y futuros licenciados en matemática-, éstos no alcanzan completamente los modos SG-SNC y AE-SNC. Esto permite argumentar la necesidad de un trabajo enfocado en las conexiones entre los modos de pensar el SNC, ya que se evidencian elementos que les permiten situarse en los modos, tales como estructura algebraica y de orden del sistema numérico, operaciones y transformaciones en el plano complejo. En síntesis, se concluye la necesidad de continuar potenciando el trabajo en los modos de pensar SG-SNC y AE-SNC, en diálogo con otros referentes teóricos, así como también en el tránsito entre los modos de pensamiento descritos en este trabajo. En particular, los modos de pensamiento y articuladores validados en este estudio aportan conocimiento para el diseño de situaciones de aprendizaje que promuevan la comprensión profunda del SNC.

\section{AGRADECIMIENTOS}

Este trabajo ha sido financiado por el programa de Formación de Capital Humano Avanzado de CONICYT, a través de la Beca Magister Nacional concurso complementario 2013. Así como también por el proyecto FONDECYT №1180468.

\section{REFERENCIAS}

Ahmad, A. W. y M. Shahrill, Improving Post-secondary Students' Algebraic Skills in the Learning of Complex Numbers; International Journal of Science and Research, 3(8), 273-279 (2014)

Artigue, M. y A. Deledicq, Quatre Étapes dans L'histoire des Nombres Complexes: Quelques Commentaires Épistémologiques et Didactiques, Cahier DIDIREM 15, IREM Paris 7, Paris, Francia, (1992)

Aznar, M. A., M. L. Distéfano, G. Prieto y E. Moler, Análisis de Errores en la Conversión de Representaciones de Números Complejos del Registro Gráfico al Algebraico, Revista Premisa, 12 (47), 13-22 (2010)

Bagni, G., La Introducción de la Historia de las Matemáticas en la Enseñanza de los Números Complejos. Una Investigación Experimental Desempeñada en la Educación Media Superior; Revista Latinoamericana de investigación en Matemática Educativa, (4)1, 45-61 (2001)

Carreño, X. y X. Cruz, Álgebra. Editorial Arrayán, Santiago (2011)

Creswell, J., Educational Research: Planning, Conducting and Evaluating Quantitative and Qualitative Research, $4^{\mathrm{a}}$ Ed. Pearson Education, Boston, USA (2012)

Danenhower, P., Teaching and Learning Complex Analysis at two British Columbia Universities, 1-298. Simon Fraser University, Canadá (2000)

Distéfano, M. L., M. A. Aznar y M. Pochulu, Errores Asociados a la Representación Geométrica-Vectorial de Números Complejos: un Análisis Ontosemiótico, Revista Iberoamericana de Educación Matemática UNIÓN, (30), 61-80 (2012)

Hamilton, W. R., Theory of Conjugate Functions, or Algebraic Couples; with a Preliminary and Elementary Essay on Algebra as the Science of Pure Time, Transactions of the Royal Irish Academy, 17(part 1), $293-422$ (1837)

Hungerford, T., Algebra: Graduate Texts in Mathematics. Springer, Estados Unidos (2010)

Karakok, G., H. Soto-Johnson y S. A. Dyben, Secondary Teachers' Conception of Various Forms of Complex Numbers, Journal of Mathematics Teacher Education, 18(4), 327-351 (2015)

Kline, M., El Pensamiento Matemático de la Antigüedad a Nuestros Días. Alianza Editorial S.A, Madrid, España (1972)

Martínez-Sierra, G. y R. Antonio, Una Construcción del Significado de Número Complejo y su Operatividad; En P. Lestón (Ed.), Acta Latinoamericana de Matemática Educativa, 22, 1033-1039 (2009) 
Saiz, O. y V. Blumenthal, Matemática $3^{\circ}$ medio, Texto para el Estudiante, Editorial Cal y Canto, Edición especial para el Ministerio de Educación de Chile, Santiago, Chile (2017)

Maumary, C. P. y M. E. Maumary, Las Tribulaciones que Generan los Números Complejos en nuestros Alumnos y en nosotros, los Docentes, IV Jornadas de Enseñanza e Investigación Educativa en el campo de las Ciencias Exactas y Naturales, La Plata, Argentina, 28, 29 y 30 de octubre (2015)

Ministerio de Educación, Objetivos Fundamentales y Contenidos Mínimos Obligatorios de la Educación Básica y Media, Gobierno de Chile, Chile (2009)

Nahin, P., An imaginary tale: The Story of $\sqrt{-1}$. Princeton University Press (1998)

Panaoura, A., I. Elia, A. Gagatsis, y G. P. Giatilis, Geometric and Algebraic Approaches in the Concept of Complex Numbers, International journal of mathematical education in science and technology, 37(6), 681-706 (2006)

Pardo, T. y B. Gómez, La enseñanza y el Aprendizaje de los Números Complejos: Un Estudio en el Nivel Universitario, PNA, 2(1), 3-15 (2007)

Parraguez, M., Teoría los Modos de Pensamiento, pp.1-80. Ediciones Instituto de Matemática de la Pontificia Universidad Católica de Valparaíso, Valparaíso, Chile (2012)

Pinto-Rojas, I. y M. Parraguez, Articulators for Thinking Modes of the Derivate from a Local Perspective, International Electronic Journal of Mathematics Education, 12(3), 873-898 (2017)

Sierpinska, A., One some Aspects of Student's thinking in Linear Algebra; On the Teaching of Linear Algebra, Dorier, J. L. (Ed.), Kluwer Academic Publishers, 209-246, Netherlands (2000)

Sierpinska, A., A. Nnadozie, y A. Oktaç, A Study of Relationships between Theoretical Thinking and high Achievement in Linear Algebra, vol 2. Concordia University (2002)

Smith, E.M., J.P. Zwolak y C.A. Manogue, Student Difficulties with Complex Numbers, PERC Proceedings, 311-314, Maryland, USA, July 29-30 (2015)

Stake, R., Investigación con Estudio de Casos. Morata, Madrid, España (2010)

Zimmermann, W. y S. Cunningham, Visualization in Teaching and Learning Mathematics. Mathematical Association of America, Washington DC, USA (1991) 
